\title{
"TRADURRE BISOGNA, NON TRADIRE!": LE PROPOSTE DI ICILIO BIANCHI IN DIFESA DELLA LINGUA ITALIANA (1939-1940)
}

\author{
Emanuele Ventura ${ }^{1}$
}

\section{ICILIO BIANCHI E LA RIVISTA «L’ALBERGO IN ITALIA»}

Il processo d'italianizzazione forzata della lingua, imposto dal regime fascista, conobbe nei primi anni ' 40 il suo momento culminante, grazie al lavoro dell'apposita Commissione per l'italianità della lingua ${ }^{2}$ (d'ora in avanti: Commissione) che, fra il 1941 e il 1943, pubblicò nel Bollettino di informarioni della Reale Accademia d'Italia (d'ora in avanti: Bol) alcuni elenchi di parole straniere accompagnate dai corrispettivi sostituti italiani ${ }^{3}$ : si tratta di un episodio molto noto della nostra storia linguistica, su cui hanno portato nuova luce alcuni contributi degli ultimi decenni, a cominciare da Raffaelli A. (2010b), che ha raccolto in un unico volume le circa duemila voci finite nelle maglie della censura, illustrando le modalità di lavoro della Commissione e i principi lessicografici che lo ispirarono ${ }^{4}$. Le decisioni del gruppo accademico godettero poi di una discreta eco nell'editoria coeva e immediatamente successiva, che si occupò di diffonderle a vari livelli della vita comune ${ }^{5}$, affidandosi a rubriche di quotidiani e riviste di larga circolazione ${ }^{6}$ : fra queste rientra anche

\footnotetext{
${ }^{1}$ Consiglio Nazionale delle Ricerche (CNR) - Opera del Vocabolario Italiano. Ringrazio Luca Serianni, Giuseppe Zarra e i revisori anonimi per la lettura di queste pagine e per le loro preziose osservazioni.

${ }^{2}$ L'istituzione della Commissione seguì, in particolare, la legge 2042 del 23 dicembre 1940, che, proibendo espressamente l'uso di termini stranieri nelle insegne pubblicitarie di tutto il territorio nazionale, costituì il provvedimento legislativo fondamentale in difesa della lingua italiana.

${ }^{3}$ I lavori dell'Accademia d'Italia, confluiti poi negli elenchi di parole straniere, erano già iniziati diversi anni addietro: «Dal 1929, anno della sua inaugurazione, l'Accademia d'Italia concentra la sua attenzione sul gergo alberghiero. Forestierismi e neologia italiana culinaria vengono affrontati nell'adunanza della classe di lettere, sin dal 1929, presenti, oltre Panzini e Marinetti, il grecista Ettore Romagnoli, Angelo Silvio Novaro, il professor Farinelli. Panzini è il precursore. Marinetti, con ben altro percorso alle spalle, ne condivide le posizioni» (Capatti, 1998: 798).

${ }^{4}$ Altri contributi di riferimento sulle politiche linguistiche del regime sono Mangoni (1974), Leso et al. (1976), Raffaelli S. (1983), Cicioni (1984), Klein (1986), Raffaelli S. (2006); più nello specifico, l'italianizzazione del lessico culinario in epoca fascista, di particolare interesse nell'ottica del presente contributo, è analizzata, sotto diverse angolature, da Quaglino (2009), Raffaelli A. (2009), Piacentini (2016); altri episodi dell'italianizzazione linguistica sono stati analizzati da Nichil (2011; 2012). Più in generale, per la lingua del fascismo, cfr. Foresti (2003), Ricci (2005), Raffaelli A. (2010a).

${ }^{5}$ Tra le pubblicazioni posteriori ai lavori della Commissione, va ricordata in particolare la 2a edizione (1943) del Barbaro dominio di Paolo Monelli: cfr. Raffaelli A. (2008: 342-343). Gli esiti della politica linguistica fascista furono, come noto, molto modesti o del tutto infruttuosi: «Il purismo xenofobo di stato ebbe [...] esiti opposti rispetto agli obiettivi iniziali: il rigetto della società italiana nei confronti di tutto ciò che era stato il movimento e il regime fascista, fece sì che non solo l'autarchia linguistica fosse bollata come una delle pagine della storia linguistica più buie della nazione, ma che, proprio per contrapporsi a questa ideologia, la permeabilità dell'italiano nei confronti dei prestiti aumentasse sensibilmente» (Piacentini, 2016: 156); si vedano anche le considerazioni di Cicioni (1984).

${ }^{6}$ «Alla stampa periodica era assegnato il compito di trasformare il dibattito da accademico in popolare e nazionale» (Capatti, Montanari, 2005: 245).
} 
il mensile, pubblicato dal Touring Club Italiano, L'albergo in Italia (1925-1943; d'ora in avanti: AINIT), sul quale Icilio Bianchi annunciava cosi ${ }^{7}$, nel fascicolo IV (7-8) del 1941 ${ }^{8}$, la prima pubblicazione di forestierismi da sostituire secondo le indicazioni della Commissione:

Con legittima soddisfazione riproduciamo gli elenchi diramati dalla Reale Accademia d'Italia. Essi, infatti, comprendono, in massima parte, vocaboli e locuzioni esotici, a sostituire i quali, fin dagli scorsi anni, abbiamo proposto in questa Rivista $[\ldots]$ vocaboli e locuzioni prettamente italiani, in gran parte accolti dalla Commissione anzidetta: si contano sulle dita di una mano le voci per le quali quest'ultima ha suggerito soluzioni diverse da quelle da noi proposte (AINIT, 1941, 7-8: 273) .

Nella sua attività divulgativa, esplicitamente indirizzata ai gestori di alberghi, trattorie e caffè (e circoscritta, dunque, agli esotismi che più riguardavano questo settore commerciale), Bianchi rammentava come il suo personale contributo all'italianizzazione fosse iniziato, in realtà, già nei fascicoli degli anni 1939 e 1940, approdando a risultati che per buona parte sarebbero poi stati accolti dalla stessa Commissione. Va infatti sin d'ora ricordato un aspetto essenziale, messo in luce da Raffaelli A. (2008), ovvero che i lavori della Commissione finirono, nel loro complesso, per essere non tanto un originale lavoro di revisione linguistica (tanto meno di vera e propria onomaturgia), ma piuttosto un'eco di proposte avanzate da singole voci, perlopiù esterne agli ambienti ufficiali ed entrate nel dibattito linguistico di quegli anni per vie limitrofe, tramite contributi lessicografici di varia provenienza (dizionari, monografie, rubriche su quotidiani e riviste nazionali) ${ }^{10}$ : esse meritano, perciò, di essere vagliate con attenzione, così da poterne anzitutto misurare l'effettiva forza d'influenza sugli elenchi successivamente pubblicati nel Bol, punto di

\footnotetext{
${ }^{7}$ Non sono moltissime le notizie biografiche sul personaggio: figlio di Augusto Guido Bianchi (cronista per il Corriere della Sera, nonché amico di Pascoli, con cui intrattenne una corrispondenza epistolare: cfr. Scarpati, 1979) fu direttore di varie riviste di inizio Novecento (fra le altre: La settimana illustrata, L'Attualita), corrispondente da Bruxelles per il Corriere della Sera, Il Piccolo e Il Messaggero, e da Milano per il francese Le Matin. Romanziere e traduttore, scrisse soprattutto gialli e libri per ragazzi (a partire dal 1907, pubblicati nella collana "Biblioteca fantastica dei giovani italiani": cfr. Pirani, 2000); fondò, nel 1919, la casa editrice Modernissima, per la quale pubblicò una biografia dell'amico Guido da Verona (1919), tra gli autori di maggiore successo degli anni Venti.

${ }^{8}$ Le altre liste si trovano nei fascicoli 9-10 e 11-12 del 1941 e nei fascicoli 5-6 e 7, 8 del 1942.

${ }^{9}$ In realtà, le proposte sostitutive nelle quali Bianchi si distanzia da quelle della Commissione sono circa la metà (cfr. infra). Nel resto della premessa, Bianchi sosteneva l'affermazione di un nuovo purismo, riconoscendo come le parole non debbano essere frutto d'imposizione accademica, e riconducendo soprattutto al trattamento del lessico tecnico il cuore della questione linguistica: «Il rispetto per la tradizione e il geloso amore per la lingua non debbono impedire l'accettazione di vocaboli nuovi per designare idee e cose nuove. I vocaboli non s'impongono per autorità di Accademie, e la massima libertà e il maggior diritto di scelta competono ai poeti, agli scrittori e, insomma, agli artisti. Ma il problema riguarda in particolare la lingua del commercio, delle finanze, delle industrie, che diciamo tecnica [...]. Non bisogna recalcitrare dinanzi a una nuova forma di purismo che rispetti la storia e accolga i termini nuovi, quando la nuova civiltà e le nuove scoperte e il progresso dello spirito lo impongono [...]. Ma talora, quando il capriccio, anziché la storia, favorisce la propagazione di una parola forestiera, è doveroso intervenire prontamente [...]. Non si vuole rinnovare un esagerato purismo, ma si vuole propugnare una forma di nuovo purismo, che sarà insieme proprietà e decoro linguistico e che rispetterà la nostra tradizione, mentre concilierà le nostre esigenze con quelle che il progresso mondiale richiede anche in fatto di lingua. Italianità di lingua e italianità di pensiero. La lingua è la Nazione» (AINIT, 1941, 7-8: 273-274).

10 Le soluzioni prodotte dalla Commissione «di norma non si possono considerare direttamente riconducibili al sodalizio accademico, quanto alla tradizione di un passato prossimo che quest'ultimo si limitava - pur autorevolmente - a ribadire» (Raffaelli A., 2008: 342). Fra gli interventi di maggiore visibilità che precedettero i lavori della Commissione, vanno ovviamente ricordati quelli accolti nella rivista Lingua Nostra, che Bruno Migliorini fondò nel 1939.
} 
convergenza e di approdo definitivo per le diverse esperienze puristiche avviate negli anni addietro ${ }^{11}$.

In questa sede, dunque, ci occuperemo del contributo apportato da Icilio Bianchi all'italianizzazione della lingua, osservando da vicino il gruppo dei suoi interventi pubblicati nell'AINIT tra il 1939 e il 1940 (fascicoli 7-8, 9-10, 11-12 del 1939; 1-2, 3-4, 910 e 11-12 del 1940). Il lavoro approntato dal Nostro, pur costituendo un tassello tra i meno noti all'interno del mosaico purista di quegli anni, palesa un certo interesse, derivante principalmente da tre aspetti:

1. l'originalità, da lui stesso rivendicata, rispetto all'operato dei suoi più illustri predecessori (in particolare i dizionari di Panzini e Monelli, ma anche quello di Mestica $^{12}$ : cfr. infra par. 2) e la posizione, espressa anch'essa a chiare lettere (cfr. supra), di anticipatore delle scelte che poi saranno fatte proprie dalla Commissione;

2. la presenza, fra le voci chiamate in causa nell'AINIT, di una ventina di forestierismi che non saranno accolti, invece, fra le liste pubblicate nel Bol;

3. la possibilità di saggiare anche qualche tentativo di rimpiazzare alcune voci regionali, che a loro volta finirono per essere oggetto di ostracismo nella propaganda linguistica di quegli anni ${ }^{13}$.

Più in generale, poi, di là dall'efficacia e dall'effettiva risonanza delle proposte avanzate, va soprattutto ricordato come tutte le esperienze analoghe di quegli anni costituiscano dei documenti significativi «non certo per il merito delle cose dette - spesso dilettantesche e impressionistiche - bensì per il loro innegabile interesse storico, per la possibilità di istituire un confronto retrospettivo (cambia, e per quali aspetti, l'atteggiamento rispetto al purismo ottocentesco?) e per una proiezione nel futuro (c'è stato qualche effetto di queste campagne nell'italiano successivo? ed è possibile accertarlo?)» (Serianni, 2011: 270).

\section{LE PROPOSTE DI ICILIO BIANCHI PER ITALIANIZZARE IL LESSICO DEGLI ALBERGHI E LA LORO "BABELICA TERMINOLOGIA CUCINARIA"}

Come anticipato, l'opera puristica del Bianchi era già iniziata nei fascicoli 7 e 8 dell'AinIt (1939): scopo dell'impresa e della sua particolare destinazione editoriale era (oltre alle riflessioni indotte dalla lettura di un articolo di Carlo Linati sul tema Lingua e autarchia, apparso nel "Giornale d'Italia") la volontà di pubblicare i risultati di alcune ricerche personali, condotte con l'intento di aiutare un amico "preoccupato di italianizzare al cento per cento le "liste" del suo albergo» (AINIT, 1939: 252); le riflessioni offerte, infatti, riguardano soprattutto il «vastissimo campo della babelica terminologia cucinaria» (ibid.), che costituirà una componente primaria (circa 300 voci $)^{14}$ anche tra le circa duemila

${ }^{11}$ Raffaelli A. (2009: 362-363) caldeggia appunto «un'indagine sulle iniziative lessicografiche coeve o di poco precedenti», in modo tale da riuscire a «quantificare il peso dei riferimenti (specie del Dizionario moderno di Panzini) della Commissione, e dunque appurarne l'originalità o piuttosto l'approccio derivativo».

12 Sul ruolo fondamentale di Panzini (oltre a quello della Scienza in cucina di P. Artusi) in quelle che saranno, invece, le scelte sostitutive della Commissione, cfr. le statistiche rilevate da Piacentini (2016: 178-179). Per il Dizionario della lingua italiana (1936) di Enrico Mestica, cfr. Fanfani (2018).

13 A tal riguardo, cfr. le parole di Carlo Formichi, vice presidente della Classe di Lettere della Reale Accademia d'Italia e presidente della Commissione: «Si rivela più italiano chi meglio parla italiano. Chi adopera un vocabolo dialettale, o, peggio, straniero invece di quello italiano, rivela non solo un difetto di cultura ma di patriottismo» (cit. in Piacentini, 2016: 151).

${ }^{14}$ Cfr. Raffaelli A. (2009: 356): «L'attenzione all'argomento culinario non sorprende, trattandosi di un ambito rilevante e prestigioso dell'attività produttiva italiana; esso inoltre rientrava nella connotazione tecnico-settoriale, vicina al mondo delle imprese e dei commerci, che caratterizzò l'intera iniziativa». 
sostituzioni complessivamente accolte dal Bol. Nella breve introduzione preposta alle prime voci licenziate, il Bianchi cita le sue principali fonti e traccia il modus operandi che lo ha guidato e che, a suo dire, dovrebbe guidare tutti gli addetti ai lavori incaricati di «liberare la lingua italiana dalla terminologia stranieras ${ }^{15}$ :

Naturalmente, abbiamo spessissimo attinto al Dižionario moderno di Alfredo Panzini e al Barbaro dominio di Paolo Monelli, ma dovremo più volte dissentire da essi, perché una dubbia conoscenza della materia, limitata, nel nostro caso, al campo della gastronomia, li ha tratti qualche volta - il primo più del secondo - a ingannevoli definizioni.

Beninteso, qui non si vuol fare del purismo alla Puoti, come lo intendono tuttora quei pedanti che, per idolatria della intangibilità della lingua, si son fatti o si fanno in quattro per imprigionare in schemi inviolabili la realtà viva $\mathrm{e}$ dinamica del nostro linguaggio $[\ldots]$.

Ma attenti a mali passi, attenti a non cascare nell'improvvisazione facilona.

Per ritornare ai termini dell'arte cucinaria, in questi ultimi mesi abbiamo avute ripetute prove del giusto tentativo di tradurre in buon italiano i nomi di pietanze che per anni e anni ci siamo ostinati a definire con parole straniere. Anche a quegli elenchi ci rifaremo, e non sempre per approvare le versioni in essi prescelte. Tradurre bisogna! Non tradire! [...] (ibid.)

Le voci elencate da Bianchi sono 136 ${ }^{16}$, di cui 90 francesismi ${ }^{17}, 22$ anglicismi, 14 germanismi e due termini di altra provenienza (il giapponesismo, di tramite francese, kaki, e il russismo zacusca). A queste si aggiungono 8 regionalismi: anguria, considerata come voce tipica di Lombardia, Veneto ed Emilia per cocomero; arzente 'acquavite', «idiotismo toscano per ardente» (AINIT, 1940, 1-2: 49); i lombardismi barbajata 'a Milano, bevanda di latte e cioccolata', per la quale si rifiuta la forma toscanizzata barbagliata («Qualcuno crede di parlare scelto usando il nome barbagliata; ma si tratta di una licenza inammissibile, derivando la parola dal nome del De Barbaja, il celebre impresario del tempo di Gioacchino Rossini» AINIT, 1939, 7-8: 252), brasato («deformazione lombarda - derivante quindi, come spesso avviene, dal francese braise [...] - della parola abraciato» AINIT, 1939, 7-8: 253) e (in) carpione («dal milanese carpion e carpionà, e sta a classificare vivande, pesci quasi sempre [...], cucinati come richiede il carpione» AINIT, 1939, 7-8: 253), al quale andrà preferito l'agg. marinato; verza, da rimpiazzare con cavolo o cavolfiore («In Lombardia e nell'alta Emilia si dice verza per cavolo» AINIT, 1940, 1-2: 50); zabaione («è ridicolo credere

${ }^{15}$ Cfr. supra, nota 8.

${ }^{16}$ Otto termini deonomastici di origine francese, con le rispettive proposte di sostituzione, sono già segnalati da Raffaelli A. (2008): béchamel, champagne, chantilly, chateaubriand, cognac, julienne, praline, salmis. Le proposte di Bianchi collimano in qualche caso con quelle di altri contributi coevi, anch'essi precedenti ai lavori della commissione: fra questi vanno ricordati, oltre a Panzini (1923) e Monelli (1933), Jàcono (1939) e Natali (1940), ma anche altri lavori dedicati a settori specifici del lessico, per i quali cfr. Raffaelli A. (2008: 340342); sugli strettissimi rapporti fra le opere di Monelli (1933) e Jàcono (1939), e sulle accuse di plagio rivolte a quest'ultimo, cfr. Serianni (2011) e Piacentini (2017). Il Monelli, poi, andrà ricordato anche per Il ghiottone errante: viaggio gastronomico attraverso l'Italia (1935), tra le prime testimonianze della critica enogastronomica italiana.

${ }^{17}$ La presenza preponderante del francese è del tutto in linea con l'epoca storica, nella quale agisce una «sorta di riflesso condizionato, innestato dalla tradizione puristica: dire forestierismo significa ancora dire francesismo» (Serianni, 2006: 65); inoltre, se gli anglicismi appaiono perlopiù come prestiti di necessità, i francesismi fanno soprattutto parte della conversazione borghese (ibid.). Va poi rammentato che «l'influenza di marca francese nella cucina italiana inizia prepotentemente a partire dal XVIII secolo: fatto che determina inevitabilmente un largo utilizzo di francesismi, adattati e non adattati, nella lingua italiana» (Piacentini, 2016: 176); il peso del francese è riconoscibile, però, già in epoca medievale (cfr. Frosini, 2012: 86): sarà poi fondamentale, a cavallo fra Ottocento e Novecento, il ruolo dell'Artusi nel «razionalizzare quel "gergo infranciosato" che invadeva i trattati di cucina del tempo» (Beccaria, 2012: 4). 
(C) Italiano LinguaDue 2. 2021. E. Ventura, “Tradurre bisogna, non tradire!”: le proposte di Icilio Bianchi in difesa della lingua italiana (1939-1940)

di ingentilirla, storpiandola in zabaglione» AINIT, 1939, 11-12: 354); in riferimento agli alcolici, poi, è considerato un malvezzo molto diffuso (destinato a imporsi fino alla lingua contemporanea) l'agg. liscio per puro.

I forestierismi (quasi sempre integrali, più raramente consistenti in forme assimilate, a dimostrazione di come l'orizzonte d'intervento sia limitato alle innovazioni recenti) sono riconducibili a tre macro-gruppi:

a) termini le cui proposte sostitutive coincidono con quelle che più tardi saranno ufficializzate dalla Commissione ${ }^{18}$;

b) termini per i quali Bianchi opera scelte di traduzione differenti rispetto alla Commissione;

c) termini assenti negli elenchi pubblicati nel Bol.

a) Il primo macro-gruppo consta di 56 parole (il 41, $17 \%$ del totale) che, oltre a testimoniare il ruolo precorritore del Nostro (o delle fonti alle quali egli talvolta attinse), consentono di tracciare alcune osservazioni generali e in parte valide anche per gli altri due gruppi. Anzitutto, si può rilevare come, in diversi casi, le soluzioni appaiano piuttosto immediate, grazie alla presenza, già a quell'epoca, di un corrispondente italiano comune o di alto uso (come frutto di adattamento o come termine indipendente dal forestierismo e con esso in un rapporto di sinonimia o di perfetta coincidenza), che connota il forestierismo come prestito di lusso: ${ }^{19}$ anisette $\rightarrow$ anisetta; beef-steak $\rightarrow$ bistecca $^{20}$; béchamel $\rightarrow$ besciamella ${ }^{21}$; breakfast $\rightarrow$ prima colazione; caramel $\rightarrow$ caramellata ${ }^{22}$; chope $\rightarrow$ boccale (di birra); concierge $\rightarrow$ portiere; confiture $\rightarrow$ marmellata; cordial (bevanda) $\rightarrow$ cordiale; cotoletta (adattamento del fr. côtelette $) \rightarrow$ costoletta; croquette $\rightarrow$ crocchetta; crème $\rightarrow$ crema; cremeria (adattamento del fr. crèmerie) $\rightarrow$ latteria; $d r y$ e extradry (spumante) $\rightarrow$ secco, strasecco (in tal caso con l'aggiunta del prefisso); fondant $\rightarrow$ fondente; giambone $\rightarrow$ prosciutto $^{23}$; lift $\rightarrow$ ascensore $^{24}$; marrons glacés $\rightarrow$ marroni canditi; menu $\rightarrow$ lista; omelette $\rightarrow$ frittata $^{25}$; restaurant $\rightarrow$ ristorante. Qualche forma è esplicitamente desunta dalla tradizione (blanc-manger $\rightarrow$ biancomangiare ${ }^{26}$; consommé $\rightarrow$ brodo ristretto/ristretto $)^{27}$, o preferita rispetto a un'altra concorrente per via di illustri ascendenze letterarie, come avviene per lattemiele (voce, peraltro, già cinquecentesca: cfr. GDLI s.v.) rispetto a panna montata, nella traduzione del francesismo chantilly («il Carducci l'ha crismato in una sua lirica» AINIT, 1939, 7-8: 253).

\footnotetext{
${ }^{18}$ Le sostituzioni pubblicate nel Bol sono tutte ricavate da Raffaelli A. (2010b).

19 O forestierismo superfluo (distinto dai forestierismi insostituibili e dai forestierismi utili), per usare la nomenclatura, più scientificamente fondata, preferita da Adamo, Della Valle (2008: 73-74).

${ }_{20}$ Bianchi suggerisce anche braciola (o bragiola).

21 «Il Panzini e il Mestica hanno accettato la libera versione dell'Artusi [...] balsamella», forma da rifiutare poiché l'etimo (da balsamo) tradisce quello del termine francese (AINIT, 1939, 7-8: 252).

22 Bol: caramellato (sost. e agg.).

23 «Coloro che spropositano dicendo giambone [...], se mai dovrebbero dire gambone, perché il vocabolo francese [...] deriva dall'italiano gamba〉 (AINIT, 1940, 1-2: 49); giambone è già forma settecentesca (Frosini, 2012: 91).

${ }^{24}$ Cfr. Monelli (1933: 80): «inutile neologismo, visto che abbiamo già fatto italiano il francese ascensore, che è del resto di pura origine latina».

25 Come ricorda Bianchi, i due termini non sono perfettamente coincidenti («i cuochi fanno qualche differenza fra omelette e frittata. La prima, più molle, a mezza cottura vien ripiegata su se stessa: di qui il nostro arcaico, ma efficace, vocabolo pesceduovo, dalla forma di pesce che assume l'omelettes): cfr. Quaglino (2009: 373) e Piacentini (2016: 167).

26 Presente già in Pietro Aretino (cfr. GDLI s.v.); cfr. anche TLIO (s.v. bramangiere).

${ }^{27}$ «Il Monelli e il Panzini consigliano consumato, parola che è nella lingua dei nostri cuochi cinquecenteschi [...]. Noi, però, come lo Scappi, illustre cuoco del '500, preferiamo brodo ristretto, od anche, più succintamente, ristretto» (AINIT, 1939, 9-10: 311).
} 
(C) Italiano LinguaDue 2. 2021. E. Ventura, “Tradurre bisogna, non tradire!”: le proposte di Icilio Bianchi in difesa della lingua italiana (1939-1940)

Altre coincidenze più significative tra Bianchi e il Bol sono: al trancio $\rightarrow$ a fette, a taglio; brûlé $\rightarrow$ caldo (vino); buffet $\rightarrow$ tavola fredda, rinfresco ${ }^{28}$; buvette $\rightarrow$ mèscita (con é chiusa nel Bol); dépendance $\rightarrow$ dipendenza; dessert $\rightarrow$ alla frutta ${ }^{29}$; drops (caramelle) $\rightarrow$ gocciole; entremets $\rightarrow$ piatti di mezzo; flan $\rightarrow$ sformato; gigot $\rightarrow$ cosciotto; Goulasch $\rightarrow$ spezzatino all'ungherese ${ }^{30}$; grape-fruit $\rightarrow$ pompelmo; gratin $\rightarrow$ (maccheroni) crostati (Bol: crostato); griglia $\rightarrow$ graticola, gratella ${ }^{31}$; grill room $\rightarrow$ rosticceria (Bol: sala di rosticceria); hors-d'oeuvre $\rightarrow$ antipasto; Kümmel $\rightarrow$ comino (Bol: cumino); Kursaal $\rightarrow$ casìno; mélange $\rightarrow$ barbajata con panna $^{32}$; (Bol: barbagliata); mousse $\rightarrow$ spumone ${ }^{33}$; mousser $\rightarrow$ spumeggiare; pâté $\rightarrow$ pasticcio; poché $\rightarrow$ in camicia; pot-au-feu $\rightarrow$ lesso in terrina (in Bol con variazione del determinante: lesso in pentola); purée $\rightarrow$ passato $^{34}$; smoking $\rightarrow$ giacchetta da sera ${ }^{35}$; tournedos $\rightarrow$ medaglioni di filetto (Bol: medaglione di carne) ${ }^{36}$. Alcune voci composte del tedesco favoriscono lo sviluppo di calchi strutturali: Butterbrot $\rightarrow$ panino al burro $^{37}$; Kirsch/Kirschwasser $\rightarrow$ acquavite di ciliegie. Ci sono, poi, anche alcuni forestierismi ai quali è concesso il lasciapassare alla luce della loro difficile traducibilità o della loro già duratura permanenza in italiano: cognac (è rigettata la forma adattata cognacche) e gli adattamenti grafici chifel (per Kìfel, ma il Bol proporrà anche cornetto) ${ }^{38}$; salmì (per salmis) e vèrmut (per vermouth). ${ }^{39}$

In qualche caso la corrispondenza fra la scelta di Bianchi e quella della Commissione è parziale, poiché una delle due parti consiglia più di un sostituto atto a rimpiazzare il medesimo forestierismo: è il caso, per Bianchi, di prestiti come cotoletta $(\rightarrow$ costola, costoletta); dépendance ( $\rightarrow$ dipendenza, succursale); drops ( $\rightarrow$ gocce, gocciole, stille); entremets $(\rightarrow \text { piatti di mezzo, intramessi })^{40}$; Kirsch/Kirschwasser $(\rightarrow$ acquavite di ciliegie, maraschino); mousser ( $\rightarrow$ spumeggiare, spumare), ecc.; d'altro canto, sebbene la Commissione tenda a privilegiare una maggiore univocità del sostituto, si può talvolta constatare anche la situazione contraria: chantilly ( $\rightarrow$ panna montata, lattemiele); chope $(\rightarrow$ gotto, boccale); confiture ( $\rightarrow$ confettura, marmellata [ma solo quando il prodotto conserva la frutta in pezzi] $)^{41}$; pâté ( $\rightarrow$ pasticcino, pasticcio); poché ( $\rightarrow$ uovo in camicia, uovo affogato).

${ }^{28}$ Nello specifico, la Commissione sceglie rinfresco (nei ricevimenti) e caffe (nelle stazioni), oltre a tavola fredda come traducente della loc. buffet freddo. Cfr. Raffaelli A. (2010b: 62 nota 59).

${ }^{29}$ Oltre alla loc. avverbiale, il Bol propone anche il sost. fin di pasto.

${ }^{30}$ Per Strudel, Goulash e altre voci provenienti dallo spazio germanofono e mitteleuropeo, va sempre tenuto in conto che, pur trattandosi di parole straniere, esse erano probabilmente ben presenti anche in alcune cucine "italofone" (Trentino, Venezia-Giulia).

31 Ampia, tra le rubriche puriste dell'epoca, è la convergenza verso questi due sostituti (cfr. Quaglino, 2009: 373). Bianchi segnala anche la loc. ai ferri, bollata però come inutile neologismo.

32 Loc. suggerita dalla "Dante Alighieri".

33 Voce artusiana (cfr. GDLI s.v. \$1 'dolce di consistenza spumosa, i cui ingredienti base, latte, zucchero e in genere cioccolato, sono amalgamati con albume montato a neve e panna montata'). Bianchi propone anche spuma; il Bol, invece, aggiunge spumata.

34 «La "Dante" consiglia anche purea, ma è parola barbara» (AINIT, 1939, 11-12: 354); sostituto già consigliato da Rigutini (1886) e Fanfani, Arlia (1890).

35 «smoking in Gran Bretagna vuol dire fumatore [...], non mai quella giacchetta che un tempo fu la smokingjacket ed oggi è senz'altro la dinner-jacket» (AINIT, 1940, 1-2: 49 s.v. dancing).

36 «non hanno nulla a che vedere coi saltimbocca, con le quagliette e gli uccelli scappati, come vorrebbe il Panzini» (AINIT, 1939, 11-12: 354).

37 «non già in panino imburrato e, tanto meno, in burro e pane, come suggerisce il Panzini» (AINIT, 1940, 3-4: $110)$.

38 Cfr. Quaglino (2009: 371).

39 «Dirai vermut e magari, quando lo offri alla tua bella, vermuttino [...]. I puristi strilleranno o, putacaso, vi chiederanno di pronunciare alla toscana vermùtte [...]» (AINIT, 1939, 7-8: 255).

40 «L'Artusi scrive tramessi; altri, con più....musicale eleganza, dicono intermezzi [...]. Quindi, se volete parlar forbitissimo, dite, se mai, intramessì (AINIT, 1939, 9-10: 311-312).

41 «Pensate al D’Annunzio che da un paggio fece versare le confetture nel seno di Giulia Farnese! Se si fosse trattato di marmellata, povera Giulia!» (la medesima considerazione è già presente in Monelli, 1933: 81). 
b); c) Di maggiore interesse appaiono, in questa sede, i 71 forestierismi restanti: 49 di essi indurranno Bianchi verso sostituzioni diverse da quelle successivamente operate dalla Commissione; gli altri 22, invece, non saranno inclusi negli elenchi del Bol (questi ultimi, come si vedrà, sono perlopiù dei termini che, pur non avendo probabilmente goduto di una reale circolazione nella lingua comune, dovevano essere di uso frequente all'interno degli alberghi e dei loro ristoranti ${ }^{42}$, soprattutto in presenza di una nutrita clientela straniera). Sarà utile offrirne un quadro sinottico, al quale alleghiamo anche, a testimonianza della revisione linguistica condotta dall'autore, alcuni brevi estratti delle note etimologiche e dei commenti puntualmente allegati da Bianchi al fine di giustificare le sostituzioni proposte o chiarire il significato del termine ${ }^{43}$ :

Tabella 1. Sostiturioni di Bianchi e della Commissione a confronto

\begin{tabular}{|c|c|c|}
\hline FORESTIERISMI & SOSTITUZIONE PROPOSTA DA BIANCHI & $\begin{array}{l}\text { SOSTITUZIONE } \\
\text { PROPOSTA } \\
\text { DALLA } \\
\text { COMMISSIONE } \\
(B o l)\end{array}$ \\
\hline $\begin{array}{l}\text { aperitif sic (anche } \\
\text { infra; AINIT 1940, 3- } \\
4 \text {, p. 110) }\end{array}$ & $\begin{array}{l}\text { aperitivo, tonico («Ormai non ci sono che i gagà ad } \\
\text { usare la parola francese }[\ldots] \text {. Invero }[\ldots] \text { si } \\
\text { dovrebbe dire tonico, perché aperitivo }[\ldots] \text { è, più } \\
\text { propriamente, bevanda che serve a dilatare i pori, a } \\
\text { promuovere le secrezioni biliari») }\end{array}$ & - \\
\hline $\begin{array}{l}\text { aspic (AINIT 1939, } \\
11-12 \text {, p. } 353)\end{array}$ & in gelatina & - \\
\hline $\begin{array}{l}\text { bar (AINIT 1939, 7-8, } \\
\text { p. 252) }\end{array}$ & $\begin{array}{l}\text { bar («I Toscani hanno mèscita, in origine soltanto } \\
\text { spaccio di vino. Questo vocabolo sarebbe } \\
\text { indubbiamente il migliore; Ma è troppo tardi per } \\
\text { dare l'ostracismo all'anglica parola ban») }\end{array}$ & - \\
\hline $\begin{array}{l}\text { bavaroise (AINIT } \\
\text { 1940, 3-4, p. 110) }\end{array}$ & $\begin{array}{l}\text { bavarese («bevanda bollente, in origine preparata con } \\
\text { tè, fior di latte, scorza di limone e sciroppo») }\end{array}$ & - \\
\hline $\begin{array}{l}\text { beignet (AINIT } \\
\text { 1939, 7-8, p. 252- } \\
\text { 253) }\end{array}$ & $\begin{array}{l}\text { bombolone («Troppo facile e disinvolta la versione } \\
\text { fonetica in bigné, ammessa anche dal Panzini e dal } \\
\text { Mestica, i quali sembrerebbero però preferire il } \\
\text { nome frittella. Ma assai diversa da questa è il beignet } \\
[\ldots] »)\end{array}$ & bignè \\
\hline $\begin{array}{l}\text { bollito (AINIT 1939, } \\
\text { 7-8, p. 255) }\end{array}$ & $\begin{array}{l}\text { lesso, allesso («è questo il nome esatto, e l'affermiamo } \\
\text { a dispetto di quanti, per amor di snobismo (questa } \\
\text { sì che è una voce straniera da importare!) si ostinano } \\
\text { a chiamare la carne lessata col francesismo bollito (da } \\
\text { boulli)! [...] Se proprio ci tenete a parlar meno } \\
\text { comune, dite patate (oppure carne, oppure pesce, } \\
\text { ecc.) allesso!») }\end{array}$ & - \\
\hline
\end{tabular}

42 A queste si accoda anche qualche parola, a cominciare da bar, che non compare nelle liste del Bol alla luce del suo profondo radicamento nella lingua comune (cfr. Raffaelli A., 2009: 358; 2010: 59).

${ }^{43}$ Non sempre le proposte etimologiche appaiono affidabili, come si vede per la voce zabaione («deriva forse dalla voce illirica zabaia, specie di bevanda» AINIT, 1939, 11-12: 354). 
(C) Italiano LinguaDue 2. 2021. E. Ventura, "Tradurre bisogna, non tradire!": le proposte di Icilio Bianchi in difesa della lingua italiana (1939-1940)

bordure (AINIT

1939, 11-12, p. 353)

bouquet (AINIT

1940, 3-4, p. 110)

brioche (AINIT

1939, 9-10, p. 311)

cabaret (AINIT

1939, 11-12, p. 353)

canapè (AINIT 1940, 3-4, p. 110)

canard (AINIT 1939, 7-8, p. 253)

carrè (AINIT 1939, $7-8$, p. 253)

champagne (AINIT 1939, 9-10, p. 311)

charlotte (AINIT 1939, 9-10, p. 311)

chateaubriand (AINIT 1939, 7-8, p. 253) guarnizione

contorno

1) mazzo, mazzetto, mazzolino; 2) aroma, fragranza («Con questa parola i Francesi intendono un insieme di fiori o di altre cose gentili [...] ed anche l'aroma del vino [...]; nel secondo, aroma o fragranza (meglio che profumo, come suggerisce il Panzini)»)

focaccetta

brioscia

vassoio, guantiera («Meglio la prima locuzione, servendo la seconda anche per la scatola in cui si tengono i guanti»)

in canapè («In cucina, la voce non sta a significare un divano [...] ma un modo di servire gli uccelli e, talvolta, le uova, adagiati su fragranti crostoni di pane»)

anatra («quando osti di pochi scrupoli vi servono pescecane per branzino o maiale per vitello, anche questi sono dei canards..., perché in francese canard vuol dire altresì frottolal»)

costolata («Con questa parola si ottiene un'esplicita distinzione dalle costolette e si chiarisce il fatto che il carrè è cucinato in un pezzo solo, non separando una costoletta dall'altra»); in AINIT (9-10, p. 311) si ricorda anche la voce arista («se si tratta di carrè di maiale»)

1) champagne (vino francese); 2) spumante (vino italiano) ("Quando si tratta di autentico vino francese, noi siamo per la parola francese. Diciamo, quindi e liberamente, champagne o, alla toscana, ma ci sembra un'inutile leziosità, sciampagna. Viceversa, nel caso di pretto vino delle nostre terre, diciamo sempre e soltanto spumantes) ${ }^{45}$

crostata (di mele, di frutta, ecc.) («Il Panzini sembra dare il passo alla barbara traduzione ciarlotta. ${ }^{46}$ Noi suggeriamo crostata (crostata di mele, oppure di frutta ecc.»)

filettone di bue («Si è proposto [...] doppio trancio di filetto di bue. Malissimo [...]. Ad ogni modo si dovrebbe dire trancia; ma è un brutto lombardismo! Perché non andare per le spicce e non chiamare lo Chateaubriand: filettone di bue?») sciampagna

carlotta

gran filetto ${ }^{47}$

\footnotetext{
${ }^{44}$ Solo nell'accezione di 'locale in cui si tengono spettacoli di varietà' (sostituto: taverna).

${ }^{45}$ La medesima distinzione si ritrova in Monelli (1933) e Jàcono (1939).

${ }^{46}$ Ciarlotta è voce adoperata anche da Artusi (cfr. Artusi, 2010: 707).

${ }^{47} \mathrm{La}$ soluzione gran filetto potrebbe aver risentito proprio della forma filettone di bue proposta da Bianchi (cfr. Raffaelli A., 2008: 343, nota 20).
} 
(C) Italiano LinguaDue 2. 2021. E. Ventura, “Tradurre bisogna, non tradire!”: le proposte di Icilio Bianchi in difesa della lingua italiana (1939-1940)

\author{
cherry-brandy \\ (AINIT 1940, 1-2, p. \\ 49)
}

choucroute (AINIT

1940, 1-2, p. 49)

civet (AINIT 1940, 1 2, p. 49)

comfort (AINIT

1939, 11-12, p. 353)

coperto (AINIT

1940, 3-4, p. 110)

coque (AINIT 1939, $9-10$, p. 311)

\author{
côtes de bœuf \\ (AINIT 1939, 9-10, p. \\ 311)
}

cotillons (AINIT

1940, 1-2, p. 49)

crêpe (AINIT 1940, 1-2, p. 49)

dancing (AINIT

1940, 1-2, p. 49)

en belle vue (AINIT 1939, 11-12, p. 353) rosolio di ciliege

ratafià di ciliegie

cavolo agro, cavolo forte («Il Petrocchi l'ha tradotto in

cavolo all'aceto salcràutte, mentre il Panzini è per la più comune versione sarcrauti. Altri usa [...] crauti. Noi preferiremmo parole più italiane»)

stufatino di lepre, cibreo di lepre

stufato di lepre

conforto, comodo, comodità, modernità («parola inglese comodità che i più, da noi, pronunziano come se appartenesse alla lingua di Francia [...]. Dirai dunque conforto o, se hai bisogno di termini diversi, comodo, comodità, modernità, ecc. [...] Invece di camera, appartamento con tutti i comforts moderni, si dica con tutte le comodità, con ogni modernitàs)

1) servito, servizio; 2) commensale («Il Rigutini suggerisce la parola tovagliolo o posata [...]. Il Panzini, invece, consiglia la parola servito $[\ldots] \mathrm{ma}$, nei casi più frequenti, si può dire servizio (percentuale per il servizio), commensale (pranzo per cinquanta commensali)»)

novo al latte («Lo Scappi [...] scrive nova da bere. A Milano si dice anche nova al latte. Il Panzini pare assolvere persino gli sciagurati che dicono nova alla coca! Noi pensiamo che la denominazione meneghina sia la migliore, poiché caratterizza il lieve imbiancamento dell'albume per la brevissima sosta dell'uovo nell'acqua a bollore»)

costole di manzo

1) doni, regali, sorprese; 2) ballo figurato ( $\Vdash$ Volendosi alludere al ballo, possiamo usare la locuzione ballo figurato»)

1) crespo, crespone; 2) focaccina, cialda, crespella ( $\mathrm{La}$ parola serve tanto a definire un notissimo tessuto di lana o di seta, quanto un dolce»)

sala da ballo («Parola inglese che, nel significato attribuitole da noi, è usata dappertutto...fuorché in Inghilterra, dove dancing è l'azione del ballare, mentre il luogo del ballo si dice dancing-room»)

in addobbo («delle pietanze preparate con arte e fantasia, in modo da appagare non soltanto il palato, ma anche la vista») 48
1) coperto;

2) persona

uovo scottato

ballo a sorpresa

créspola, cencio, frappa

sala di danze sala di danze 
(C) Italiano LinguaDue 2. 2021. E. Ventura, "Tradurre bisogna, non tradire!": le proposte di Icilio Bianchi in difesa della lingua italiana (1939-1940)

en boîte (AINIT

1939, 11-12, p. 353)

en croûte (AINIT

1940, 3-4, p. 110)

entrecôte (AINIT

1939, 9-10, p. 311)

entrée (AINIT 1939, $9-10$, p. 311)

five o' clock (AINIT 1939, 11-12, p. 353)

frappé (AINIT 1939, $9-10$, p. 312)

fricandeau (AINIT 1939, 9-10, p. 312)

fumoir (AINIT 1939, 11-12, p. 353)

garage (AINIT 1940, $1-2$, p. 49)

gateaux sic (anche infra; AINIT 1939, 7 8, p. 254) in casseruola

crostato, in crosta («Locuzione francese per indicare un pasticcio (o sformato) cotto in una pasta che in forno si sia trasformata in una bella e saporita crosta di color oro»)

costata alla fiorentina («In un opuscolo diffuso dalla "Dante Alighieri" al tempo delle sanzioni si consiglia la traduzione braciola: no, la braciola è un'altra cosa, cioè una fetta di carne senz'osso»)

piatto di entrata («Non serve la panziniana versione portata [...]. Meglio tradurre letteralmente in piatto di entrata»)

merenda, al tè, all'ora del tè («Se, adunque, non vogliamo usare l'italianissimo vocabolo merenda, diciamo al tè, all'ora del tè, ecc. Così alla locuzione tearoom sostituiamo senz'altro l'espressione sala da tè [...]. È bene avvertire che si deve dire e scrivere soltanto tè, dal latino thea dei nostri naturalisti»)

1) in gelo, gelato (di spumante o altra bibita lasciata in ghiaccio); 2) latte sbattuto, caffe sbattuto (di bibita sbattuta con ghiaccio a formare una schiuma) («siccome c'è la possibilità di essere fraintesi, questo è uno dei rari casi in cui crederemmo di non dover dare l'ostracismo assoluto alla parola francese»)

noce di vitello («Il Panzini afferma che questa parola francese è stata accolta nell'italiano fricandò $[\ldots] .{ }^{49}$ Egli suggerisce anche bracinola e intingolo di vitello [...]; più esattamente, è la nostra noce di vitello»)

stanza per fumare, sala per fumatori, fumatorio («si traduce bene in stanza per fumare, sala per fumatori. Tuttavia, come si dice parlatorio, si dovrebbe dire fumatorio; parola, questa, che non è certo men bella di fumoir, di smoking-room, di Rauchsalon»)

autorimessa

1) ghiacciato;

2) frullato

arrosto (di vitello) con sugo

sala da fumo

rimessa

dolce

pasticcini («il "Giornale dei Pasticceri e Confettieri” suggeriva questa traduzione: "Dolci di crema al

crostone

(s.v. crồte)

bistecca senz'osso

tè burro". No: tutti i pasticcini, non soltanto quelli di crema, sono gateaux. Il Panzini e il Monelli propongono la parola dolci; ma essa ha un significato troppo generico»)

apposte da Bianchi, il riferimento è piuttosto alla presentazione del piatto, che era anche definita, sulla base del francese en belle vue, in bella vista: in Artusi leggiamo, ad es., la ricetta delle scaloppe di lingua farsite in bella vista («derivata dal francese (en) Bellevue, espressione che designava sprobabilmente il castello costruito per Mme de Pompadour nel $1748 \rightarrow[\ldots]$. In italiano, s̀̀ la Belle vue $\rightarrow$ è attestato dal $1773[\ldots]$; successivamente, in bella vista si ritrova in alternanza con l'altra traduzione, al belvederes) Artusi, 2010: 376).

${ }^{49}$ Forma usata da Artusi (cfr. Artusi, 2010: 286). 
(C) Italiano LinguaDue 2. 2021. E. Ventura, “Tradurre bisogna, non tradire!”: le proposte di Icilio Bianchi in difesa della lingua italiana (1939-1940)

gin (AINIT 1940, 3-4, gin («per non confonderlo con l'essenza estratta p. 111) unicamente dalle bacche anzidette [scil:: di ginepro],

gineprella

glacé (AINIT 1939, $7-8$, p. 254) per la quale useremo la voce gineprino»)

in gelatina («I francesi hanno un unico aggettivo per esprimere più qualità [...]. Con l'italiano possiamo invece sbizzarrirci. In cucina, però, glacé sta quasi sempre per in gelatina»)

habitué (AINIT

1940, 1-2, p. 49)

cliente, assiduo, frequentatore, avventore («parola, che suona male anche in francese (gli abituati?)»)

hall (AINIT 1940, 1 2, p. 49)

atrio («Parola inglese (essendo di genere neutro, è errato dire la hall, piuttosto che lo hall), che ha la sua esatta traduzione in atrio. Qualcuno propone anche aula $[\ldots] »)$

homard (AINIT

aragosta

vellutato (nell'uso gastronomico); candito, caramellato (nell'uso dolciario) 1940, 3-4, p. 111)

julienne (AINIT 1939, 7-8, p. 254)

zuppa di legumi minuti («poiché la composizione della Julienne varia col variare delle stagioni e dei luoghi, nessuno vi impedisce di chiamarla, a piacere, $Z$ uppa primaverile, Zuppa vegetariana, ecc.»)

kaki (AINIT 1940, 1- loto 2, p. 50)

Krapfen (AINIT 1939, 9-10, p. 312)

Krapfen («Non è proprio una frittella [...]. Krapfen è una di quelle parole che si potrebbe fare a meno di tradurre»)

lunch (AINIT 1939, 9-10, p. 312)

colarione («talora usata nella forma luncheon [...]. Non c'è che da adottare la corrispondente parola colazione») $)^{50}$

marbré (AINIT 1939, galantina (di lepre, di selvaggina, ecc.) («Parola francese 11-12, p. 353) che significa marmorizzato e che i salumai lombardi storpiano spessissimo in mambrè)

mayonnaise (AINIT 1939, 9-10, p. 312)

maionesa, maionese («Il Panzini ammette le traduzioni [...] maionesa o maionese. Ai lettori la scelta fra questi barbarismi»)

noisette (AINIT 1939, 11-12, p. 353)

nocciolina, nocella di vitello («Non si capisce [...] perché si sia proposto di tradurre noisettes de veau in nocettine di vitello. Se mai, dovrà dirsi noccioline $\mathrm{o}$, meglio, perché un po' di eleganza non nuoce mai, nocelle») ${ }^{51}$

office (AINIT 1939, dispensa («In qualche albergo di lusso si ha ancora il malvezzo di chiamare office quella stanza dove si dispone tutto il necessario al servizio di mensa. La parola italiana più giusta [...] sarebbe credenza; ma poiché è invalso l'uso di nominare credenza l'armadio di cucina o della sala da pranzo, diremo

sala di soggiorno, salone

àstaco

brodo alla giardiniera

cachi

bombolone, bomba

seconda colazione

marmorato dispensa»)

officio (parola schiettamente italiana per $\mathrm{i}$ servizi della mensa)

${ }^{50}$ Cfr. l'ironico commento del Novelli cit. in Quaglino (2009: 369): «gli italiani, più sobri, non hanno bisogno, come il "popolo dei cinque pasti", di mettersi a tavola due volte prima di mezzodì».

51 Cfr. s.v. fricandeau, tradotta con noce di vitello. 
(C) Italiano LinguaDue 2. 2021. E. Ventura, "Tradurre bisogna, non tradire!": le proposte di Icilio Bianchi in difesa della lingua italiana (1939-1940)

page (AINIT 1939, 11-12, p. 354)

peppermint (AINIT 1940, 3-4, p. 111)

potage (AINIT 1939, 11-12, p. 354)

poularde (AINIT 1939, 11-12, p. 354)

praline (AINIT 1939, 11-12, p. 354)

pudding (AINIT 1939, 7-8, p. 255)

roast-beef (AINIT 1939, 11-12, p. 354)

Rostbraten (AINIT 1939, 11-12, p. 354)

ragoût (AINIT 1939, $11-12$, p. 354) $)^{52}$ paggio («Gli Spagnoli chiamano botones (bottoni) questi ragazzi dalle uniformi abbottonatissime, che oziano per ore ed ore dinanzi alle porte degli ascensori o dietro alle bussole d'ingresso; gli inglesi li chiamano boys o lift-boys»)

menta piperita

menta piperita

minestra («poiché a caratterizzare la minestra densa [...] c'è in italiano la parola minestrone, diremo minestra e non potage; tanto meno, come vorrebbero alcuni, potaggio, pottaggio, potacchio [...]. Zuppa, per minestra, è un francesismo»

pollanca, pollastrina («La differenza fra poularde e pollanca è soltanto di prezzo: quando, in una lista, la nostrana pollanca si camuffa nella francese poularde potete star sicuri che il costo della porzione vi sarà almeno raddoppiato. E poiché taluni [...] confondono la pollanca con la tacchina giovane, se volete evitarvi sorprese, dite senz'altro pollastrina»)

mandorla tostata ( $(\mathrm{DDa}$ notare però che in Francia la parola pralines oggi sta a significare ogni sorta di cioccolatini ripieni, con o senza mandorla, con liquore, con crema, ecc.»)

1) budino (salato); 2) pudding (dolce) («C’è chi lo traduce in budino o bodino [...]. Ma in Francia il boudin è un budello empito di sangue e grasso di porco (il nostro sanguinaccio) [...] e non già, come il pudding, un dolce [...]. Ma il classico pudding chiamiamolo col suo nome inglese. Che diremmo se gli inglesi chiamassero big loaf il Panettone?»)

1) "alla bolognese"; 2) stufato, cibreo («La "Dante" suggerisce la parola intingolo; ma è troppo generica. Meglio usare, quando si parla di tagliatelle, maccheroni, ecc., la locuzione "alla bolognese", e quando si tratta di carne, secondo i casi, stufato, cibreo, ecc.»)

bue arrostito («Voce inglese che significa, né più né meno, bue arrostito. Potendolo chiamare così, oppure arrosto di bue, non vediamo perché si dovrebbe concedere il diritto di passaggio alla parola rosbiffe, ammessa peraltro anche dal Petrocchi, sempre disposto ad autenticare certe buffe licenze linguistiche dei Toscanil»)

braciola di maño in graticola
2) budino di frutta

1) ragì; 2) stufato

pralina

pollastro$$
\text { pralina }
$$

2) bucho di frutta

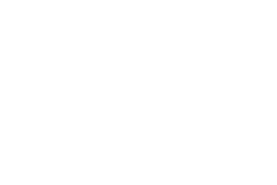

1)

$-$

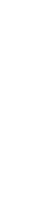

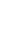


(C) Italiano LinguaDue 2. 2021. E. Ventura, “Tradurre bisogna, non tradire!”: le proposte di Icilio Bianchi in difesa della lingua italiana (1939-1940)

$$
\begin{aligned}
& \text { salvietta (AINIT } \\
& \text { 1940, 3-4, p. } 111 \text { s.v. } \\
& \text { tovagliolo) }
\end{aligned}
$$

sandwich (AINIT

1940, 1-2, p. 50)

sauté (AINIT 1940,34, p. 111)

Strudel (AINIT 1940, 3-4, p. 111)

timbale 1940, 3-4, p. 111)

(AINIT

toast (AINIT 1940, 1 2, p. 50)

toilette (AINIT 1940, $1-2$, p. 50)

vol-au-vent (AINIT $1939,7-8$, p. 255) tovagliolo

panino ripieno, tramezzino («Tartina è un francesismo (da tarte) che anche in Francia non indica il sandwich [...]. Bruttissima locuzione: panino gravido»)

rosolato, saltato («dicesi di pietanza fatta cuocere a fuoco molto vivo per brevissimo tempo [...]. Per la verità, i Francesi fanno differenza fra la pietanza fritta, sautée e rissolée. Quindi, per maggiore precisione, potremo anche noi usare, secondo i casi, rosolato $[. .$.$] e, a dispetto dei puristi...integrali,$ saltato»)

rocchio di mele («Il Panzini osserva che la voce tedesca letteralmente significa vortice o gorgo...e si dimentica di suggerirne la versione italiana. Qualcuno usa struccolo e stroccolo (di mele); ma trattasi di vocaboli che non sappiamo proprio dove siano stati racimolatil») $)^{54}$

1) sformato, pasticcio; 2) forma, stampo («La "Dante" suggerisce la corrispondente voce italiana timballo; ma questa in lingua nostra è sinonimo di timpano. Diremo, invece, sformato o pasticcio, per la vivanda; forma $\mathrm{o}$, meglio, stampo, per l'arnese di cucina in cui essa viene cotta e che gli dà la forma»)

crostino, fetta di pan tostato («Parola inglese che vuol dire fetta di pane abbrustolito e nello stesso tempo, brindisi [...]. A proposito di brindisi, i puristi ce l'hanno con questa parola, perché derivante dal tedesco bring dir's, e ti mettono innanzi l'antico verbo italiano propinare [...]. Il Panzini [...] taglia il male in mezzo e propone bere alla salute»)

ritirata («Latrina è parola che l'uso ha reso troppo volgare; lo stesso dicasi di cesso. In quanto al logo comodo dei toscani, via, ce ne sono troppi di luoghi comodi $[. .$.$] . La lingua classica ha agiamento e privato$ [...], ma son voci troppo disusate. Ė un errore, poi, adoperare la parola gabinetto (dal francese cabinet)》) $)^{56}$

sfogliatina (di carne, di rigaglie, di crostacei, ecc.) («Il nome caratterizza la lievità della pasta. Possiamo benissimo tradurre in sfogliatine (fredde o calde) $d i$ carne, oppure di rigaglie, oppure di crostacei. Il cuoco [...] può, del resto, inventare un appellativo a piacer suo, facendo seguire alla parola sfogliatina il suo

timballo

pantosto

toletta, lavabo

ventivolo

\footnotetext{
53 È riportata solo l'accezione calcistica (da sostituire con stretta).

54 Già Jacono (1939) proponeva rocchio, oltre a involto.

${ }_{55}$ Tra le voci la cui sostituzione non è ritenuta opportuna (cfr. Raffaelli A., 2010b: 36).

${ }_{56}$ Cfr. GDLI (s.v. ritirata 87: prima attestazione registrata in Carena, ante 1859).
} 
nome, o quello del suo albergo [...], ovvero chiamando di Nettuno le sfogliatine di pesce, del buongustaio quelle di carne, del ghiottone quelle di rigaglie ecc.»)

wafer (AINIT 1940, 3-4, p. 111)

cialda ripiena («la usiamo per definire uno strato di crema o di cioccolata fra due cialde»)

Würstel/Würstchen salsiccia di Francoforte («Il Sindacato dei Pubblici (AINIT 1940, 3-4, p. Esercizi di Milano ha proposto [...] salsicciotto; la 111)

"Dante" [...] salamino o salsiccetta. Ma non è chi non veda come queste nostre voci italiane manchino di esattezza e si prèstino alla confusione. È uno dei casi in cui noi saremmo tentati di proporre l'uso del vocabolo tedesco, se non si opponesse al proposito lo scempio che già se ne fa, nella pronunzia e nell'ortografia. I tipi più conosciuti di Würstel sono quelli di Francoforte e di Vienna; ma in Italia è quasi esclusivamente diffuso il primo »)

zacusca $^{57} \quad$ (AINIT

antipasto vario («Nessuna ragione, quindi, di usare la 1940, 3-4, p. 111) corrispondente parola russa»)

Zwieback (AINIT pane biscottato («non già, come vorrebbe il Panzini biscotto della salute 1940, 1-2, p. 50) [...], biscotto e, tanto meno, cantuccio»)

\section{STRATEGIE DI SOSTITUZIONE}

Raffaelli A. (2009) ${ }^{58}$ riconduce le sostituzioni proposte dalla Commissione a sei distinte categorie che, pur da non intendere troppo rigidamente (il loro confine, infatti, appare in certi casi piuttosto labile), risultano utili al fine di perlustrare le scelte puriste di Bianchi e le principali strategie da lui messe in atto per la sostituzione dei forestierismi ${ }^{59}$. In un computo complessivo che tenga conto di tutti i sostituenti (anche quelli trattati rapidamente nel macro-gruppo a), le sei tipologie si distribuiscono secondo le seguenti percentuali d'impiego: I) adattamenti grafici alle consuetudini dell'italiano: 2 [1,09\%]; II) adattamenti grafici basati sulla pronuncia italiana: 2 [1,09\%]; III) adattamenti morfofonetici $^{60} 20$ [10,92\%]; IV) riproduzioni semantiche ${ }^{61}: 106$ [57,92\%]; v) perifrasi descrittive $^{62}: 51$ [27,86\%]; VI) sostituti estrosi: $2[1,09 \%]^{63}$.

Osserviamo più da vicino le soluzioni incontrate per i macro-gruppi b) e c), quasi tutte riconducibili alle tre categorie principali (III, IV e V):

\footnotetext{
${ }^{57}$ È il russo закycka (assortimento di antipasti freddi o caldi serviti prima del pasto principale).

${ }^{58}$ Cfr. anche Piacentini (2016: 154-155).

59 Si riportano separatamente, se afferenti a gruppi diversi, le varie sostituzioni proposte al posto di un medesimo forestierismo.

${ }^{60}$ Con modifica della vocale o della desinenza finale, oppure con modifiche consonantiche e della radice.

${ }^{61}$ Facciamo rientrare in questo gruppo anche i pochi calchi semantici, cioè bavarese, conforto, in crosta, nocciolina, paggio, saltato. Per in crosta va segnalato che, di là dal legame moderno col corrispettivo fr. en croûte, la locuzione si ritrova già in epoca medievale (cfr. TLIO s.v. crosta \$2.2.1 e GDLI s.v. crosta \9); per saltato (fr. sauté), invece, la prima e unica attestazione registrata dal GDLI (s.v. \ 33 'far rosolare un cibo') è molto recente e appartiene a un articolo apparso sul quotidiano La Stampa nel 1986.

${ }^{62}$ Che «non hanno un legame semanticamente motivato con l'unità lessicale del prestito - o nelle quali comunque esso è subordinato ai tratti distintivi della specialità» (Raffaelli A., 2009: 360).

${ }^{63} \mathrm{Si}$ contano sei casi di conservazione del forestierismo.
} 
(C) Italiano LinguaDue 2. 2021. E. Ventura, “Tradurre bisogna, non tradire!”: le proposte di Icilio Bianchi in difesa della lingua italiana (1939-1940)

III) ADATTAMENTI MORFOFONETICI: aperitif $\rightarrow$ aperitivo; bavaroise $\rightarrow$ bavarese; en croûte $\rightarrow$ crostato [con ulteriore passaggio di ruolo sintattico da locuzione ad aggettivo]; fumoir $\rightarrow$ fumatorio; mayonnaise $\rightarrow$ maionesa/maionese; poularde $\rightarrow$ pollanca, pollastrina.

IV) RIPRODUZIONI SEMANTICHE: aperitif $\rightarrow$ tonico; beignet $\rightarrow$ bombolone; bollito $\rightarrow$ lesso, allesso; bordure $\rightarrow$ guarnizione; bouquet $\rightarrow 1$ ) mazzo, mazzetto, mazzolino 2) aroma, fragranza; brioche $\rightarrow$ focaccetta; buffet $\rightarrow$ rinfresco; cabaret $\rightarrow$ vassoio, guantiera; canard $\rightarrow$ anatra; champagne $\rightarrow$ spumante (vino italiano); charlotte $\rightarrow$ crostata (di...); choucroute $\rightarrow$ cavolo agro, cavolo forte; civet $^{64} \rightarrow$ stufatino di lepre, cibreo di lepre; comfort $\rightarrow$ conforto, comodo, comodità, modernità; coperto $\rightarrow 1$ ) servito, servizio 2) commensale; côtes de bouf $\rightarrow$ costole di manzo; cotillons $\rightarrow$ doni, regali, sorprese; crêpe $\rightarrow 1$ ) crespo, crespone 2) focaccina, cialda, crespella; en boite $\rightarrow$ in casseruola; five o' clock $\rightarrow$ merenda; frappé $\rightarrow$ 1) in gelo, gelato (di bibita lasciata in ghiaccio); garage $\rightarrow$ autorimessa; gateaux $\rightarrow$ pasticcini; habitué $\rightarrow$ cliente, assiduo, frequentatore, avventore; hall $\rightarrow$ atrio; homard $\rightarrow$ aragosta; kaki $\rightarrow$ loto; lunch $\rightarrow$ colazione; marbré $\rightarrow$ galantina; noisette (di vitello) $\rightarrow$ nocciolina, nocella; office $\rightarrow$ dispensa; page $\rightarrow$ paggio; peppermint $\rightarrow$ menta piperita; potage $\rightarrow$ minestra; pudding $\rightarrow 1$ ) budino (salato); ragoût $\rightarrow 2$ ) stufato, cibreo; roast-beef $\rightarrow$ bue arrostito; salvietta $\rightarrow$ tovagliolo; sauté $\rightarrow$ saltato, rosolato; timbale $\rightarrow 1$ ) sformato, pasticcio; 2) forma, stampo; toast $\rightarrow$ crostino; toilette $\rightarrow$ ritirata

V) PERIFRASI DESCRITTIVE: à la coque $\rightarrow$ uovo al latte; aspic $\rightarrow$ in gelatina; buffet $\rightarrow$ tavola fredda; chateaubriand $\rightarrow$ filettone di bue; cherry-brandy $\rightarrow$ rosolio di ciliegie; cotillons $\rightarrow$ ballo figurato; dancing $\rightarrow$ sala da ballo; en belle vue $\rightarrow$ in addobbo; entrecôte $\rightarrow$ costata alla fiorentina; entrée $\rightarrow$ piatto di entrata; five o' clock $\rightarrow$ al tè, all'ora del tè; frappé $\rightarrow 2$ ) latte sbattuto, caffè sbattuto ${ }^{65}$; fricandeau $\rightarrow$ noce di vitello; fumoir $\rightarrow$ stanza per fumare, sala per fumatori; glacé $\rightarrow$ in gelatina; julienne $\rightarrow$ zuppa di legumi minuti; praline $\rightarrow$ mandorla tostata ${ }^{66}$; ragoût $\rightarrow 1$ ) alla bolognese; Rostbraten $\rightarrow$ braciola di manzo in graticola; sandwich $\rightarrow$ panino ripieno; Strudel $\rightarrow$ rocchio di mele; toast $\rightarrow$ fetta di pan tostato; vol-au-vent $\rightarrow$ sfogliatina (di carne, di rigaglie, di crostacei, ecc.); wafer $\rightarrow$ cialda ripiena; Würstel $\rightarrow$ salsiccia di Francoforte; zacusca $\rightarrow$ antipasto vario; Zwieback $\rightarrow$ pane biscottato

VI) SOSTITUTI ESTROSI: carrè $\rightarrow$ costolata; sandwich $\rightarrow$ tramezzino

Alcuni dei forestierismi inclusi da Bianchi fra gli elenchi dell'AinIt costituiscono probabilmente una valida testimonianza della generale deferenza al modello culinario e linguistico francese, che doveva essere ancora ben visibile, in quegli anni, nel lessico alberghiero e nelle liste dei ristoranti nostrani ${ }^{67}$ : certe voci straniere, infatti, sono chiaramente ascrivibili al gruppo dei più spiccati forestierismi di lusso, la cui accoglienza in italiano fu verosimilmente effimera, e che già a quell'epoca possedevano un corrispettivo del tutto coincidente e ampiamente in uso nella lingua comune, finendo così per essere oggetto di una facile sostituzione (canard, côtes de bauf, homard, ecc.).

Il progetto di autarchia linguistica promosso da Bianchi rifugge, nel complesso, le istanze puristiche più radicali (distanziandosi esplicitamente dal purismo ottocentesco e

\footnotetext{
${ }^{64}$ Sottinteso il determinante de lièrre, de lapin, ecc.

${ }^{65} \mathrm{Il}$ traducente caffe sbattuto è già proposto da Monelli (1933).

${ }^{66}$ La loc. è adottata da Artusi (cfr. Artusi, 2010: 795).

${ }^{67}$ Una deferenza destinata in larga parte a scomparire proprio nel corso del Novecento, un secolo in cui, come è noto, proprio il lessico culinario farà da volano principale alla diffusione dell'italiano nel mondo.
} 
dai suoi principali repertori lessicografici $)^{68}$, come dimostra anzitutto l'accoglienza, in forma integrale, di alcuni prestiti ((in) canapè, champagne, gin, Krapfen ${ }^{69}$, pudding $^{70}$ ) che, nella revisione condotta dalla Commissione, saranno invece rimpiazzati per mezzo di riproduzioni semantiche: una scelta di apertura, quella ammessa da Bianchi, che si rileverà in larga parte vincente, anche alla luce dei referenti estremamente specifici qui chiamati in causa. Di contro, il Nostro sembra palesare una minore propensione agli adattamenti: sono del tutto assenti, infatti, per le voci dei macro-gruppi b) e c), gli adattamenti di tipo grafico (tanto alle consuetudini dell'italiano [il tipo vafer per wafer] quanto alla sua pronuncia [il tipo beignet per bignè]; cfr., però, nel macro-gruppo a, i casi di chifel, salmì e vèrmut), mentre quelli morfofonetici restano perlopiù circoscritti alle forme già appartenenti all'italiano in altre accezioni (calchi semantici), o che vi erano ormai entrate in modo più o meno stabile da un tempo sufficientemente ampio, rendendo così poco praticabili i tentativi di una loro estirpazione (bavaroise $\rightarrow$ bavarese $^{71}$; en croûte $\rightarrow$ in crosta, crostato; mayonnaise $\rightarrow$ maionesa/maionese ${ }^{72}$; page $\rightarrow$ paggio $^{73}$; sauté $\rightarrow$ saltato $)^{74}$. Tanto più risultano oggetto di censura quegli adattamenti morfofonetici che Bianchi bolla come forme di diffusione regionale toscana, quali consumè, mussare, rosbiffe, sciampagna (sostituzione che sarà avanzata anche dalla Commissione $)^{75}$, vermutte.

Come si evince chiaramente dall'elenco stilato supra, la strategia di sostituzione di gran lunga più adottata si fonda piuttosto sul ricorso a un corrispettivo vivo nella lingua comune (in tutti i frangenti, ovviamente, in cui non si abbia a che fare con un ritrovato recente, o specifico di una determinata realtà di provenienza), anche se ciò comporta, in qualche caso, un'inevitabile estensione semantica del traducente ${ }^{76}$, talvolta piuttosto ampia (cfr., ad es., il caso dell'anglicismo drops, i cui traducenti più o meno letterali, gocce, gocciole, stille, sono piegati ad abbracciare un referente metaforico sconosciuto all'italiano). Le riproduzioni semantiche sono preferite da Bianchi laddove gli adattamenti italiani gli appaiano, verosimilmente, ancora privi di un'esistenza pienamente codificata: sarà soprattutto il caso di brioscia, carlotta, pralina e timballo, così come degli adattamenti grafici cachi, vâfer (ma anche dei ben più antichi bignè e ragù), che saranno di lì a poco proposti dalla Commissione nel Bol; d'altro canto, si registrano anche casi di forestierismi adattati e di più lunga data ai quali, però, il Bianchi non concede il diritto di cittadinanza, come

${ }^{68}$ Cfr. supra (par. 2) il richiamo a Puoti, cui si aggiungono quelli saltuari, interni ai lemmi trattati, a Rigutini e Fanfani, per il quale si vedano infra (nota 88) le considerazioni riguardo alla voce Festival.

${ }^{69}$ Sull'etimologia di Krapfen cfr. Gheno (2020); il termine tedesco è adottato già da Artusi (cfr. Artusi, 2010: 553).

${ }^{70}$ Né Bianchi né la Commissione fanno menzione dell'adattamento morfofonetico puddingo, che compare già nel Settecento (cfr. GDLI s.v.: prima attestazione registrata in Francesco Algarotti).

${ }^{71}$ L'accezione cui si riferisce Bianchi ('bevanda di latte caldo e cioccolata') è solo una delle tre che venivano riferite al termine nell'Ottocento; cfr. Artusi (2010: 681, nota 57): «Se o come la Bavarese sia venuta dalla Baviera a Parigi, o come sia nata a Parigi con quel toponimo, non lo si sa [...]. Il termine bavarese è in Italia, alla fine dell'ottocento, polisemico e ne esistono almeno tre significati correnti, come bibita calda [...], come sformato freddo di crema, e come crema fredda, ed è questa la vincente».

72 Voce attestata già nel 1790, all'interno della loc. alla maionese (cfr. DI, III: 67).

${ }^{73} \mathrm{Nel}$ suo significato originario si tratta ovviamente di un francesismo di epoca medievale. La sostituzione page $\rightarrow$ paggio è già in Monelli (1933).

${ }_{74}$ Considerabile tra questi anche poularde $\rightarrow$ pollanca, pollastrina (cfr. Raffaelli A., 2009: 360). Per i casi di coincidenza fra Bianchi e la Commissione, cfr. anche gli adattamenti anisetta, bistecca, comino, besciamella, cordiale, crema, crocchetta, dipendenza, fondente e ristorante.

${ }^{75} \mathrm{Ma}$ sciampagna è già voce del Settecento (in S. Maffei: GDLI s.v.), e poteva oltretutto contare sull'analogo adattamento usato per designare la regione francese.

${ }^{76}$ Cfr. Raffaelli A. (2010: 64). 
avviene per bollito ${ }^{77}$, coperto ${ }^{78}$, salvietta (dei tre solo coperto, infatti, sarà oggetto di sostituzione nel Bol. In gioco, evidentemente, c'è anche la volontà di evitare delle insidiose neosemie: un esempio paradigmatico della condanna di certe dilatazioni semantiche è offerto, per es., dalla voce crema, che non solo è stata, a suo dire, erroneamente impiegata come traducente del fr. purée («Il Panzini si batte per crema; ma è un ovvio errore, perché la crema di piselli, di patate, di fagiuoli è una minestra, non un contorno» AINIT, 1939, 11-12: 354), ma che ha anche assunto l'accezione, ancor più invisa al Nostro, di 'pomata' («Siccome la crema è roba che si mangia, le signore ci usino il garbo di chiamare pomata quel miscuglio eterogeneo che si mettono sul viso» AINIT, 1939, 7-8: 254); analogamente, è ricusato l'adattamento timballo (per il fr. timbale), in quanto il termine italiano sta già a indicare lo strumento musicale del 'timpano'. In altri casi, ad ogni modo, proprio il ricorso a neologismi semantici rende aggirabili i forestierismi, come si vede, per es., nei sost. bavarese 'bevanda di latte caldo e cioccolata', tonico 'aperitivo' (in luogo del fr. aperitiff), paggio 'facchino' e 'ascensorista' di un albergo (in luogo del fr. page), o anche nelle collocazioni noccioline o nocelle (di vitello) (adattamento del fr. noisette de veau), medaglioni di filetto (per il fr. tournedos) ${ }^{80}$, rocchio di mele (per il ted. Strude), dove l'aggiunta del determinante è necessaria per rendere il traducente sufficientemente comprensibile ${ }^{81}$.

Non è praticamente contemplato il ricorso alle vere e proprie neoformazioni, se si eccettuano il termine costolata (fr. carre), peraltro di carattere derivativo (Zingarelli, 2022 offre, per carrè, la definizione di 'lombata'), e soprattutto la celebre coniazione dannunziana tramezzino (cui Bianchi accosta, però, la perifrasi descrittiva panino ripieno); i sostituti estrosi non mancheranno, al contrario, fra gli elenchi della Commissione: si pensi allo stravagante Arlecchino in luogo di cocktail (assente, però, fra le liste dell'AINIT), o anche, quanto alla voci confrontabili tra le due fonti, all'anglicismo gin, che la Commissione sostituirà con gineprella, o ancora al ventivolo, che avrebbe dovuto soppiantare il francesismo vol-au-vent. Si scorge, inoltre, il ricorso a qualche parola afferente al lessico più scelto, come nel caso di intramessi (per entremets), voce già trecentesca (cfr. GDLI s.v. intramesso ${ }^{2}$ e TLIO s.v. intramesso, da cui la parola risulta essere, per il Trecento, un hapax del Boccaccio), o di stille (per drops); di contro, anche voci popolari o regionalmente connotate possono fungere, in qualche frangente, da efficaci sostituti (cfr. il fiorentino bombolone ${ }^{82}$ per beignet, il toscano cibreo per civet e ragoût, mèscita per buvette; il lombardismo lattemiele per chantilly e i milanesismi barbajata con panna per mélange e uova al latte per à la coque).

Il tentativo di conservare un rapporto semantico quanto più possibile univoco e preciso col referente rende poco attraente la scelta di sostituti antonomastici, le cui uniche testimonianze sono offerte da costata alla fiorentina, salsiccia di Francoforte, spezzatino all'ungherese (quest'ultimo sarà accolto dalla Commissione), rispettivamente per il fr. entrecôte e per le due voci tedesche Würstel e Goulasch (adattamento dell'ungherese gulyás): appare di particolare interesse la prima perché, come visto supra, sulla perifrasi antonomastica alternativa salsiccia di Vienna, sconsigliata da Bianchi, cadrà proprio la scelta operata dalla Commissione.

\footnotetext{
${ }^{77}$ Cfr. DELI: 230: «solo il bollito di carne riproduce il fr. bouillii); la prima attestazione registrata dal GDLI appartiene a Goldoni.

${ }^{78}$ Già segnalato da Fanfani, Arlia (1877 s.v.), che costituisce a sua volta la prima testimonianza registrata dal GDLI.

${ }^{79} \mathrm{Cfr}$. già l'accezione di 'sostanza, bevanda o farmaco corroborante, ricostituente $\mathrm{o}$, anche, che ha proprietà digestive' (GDLI s.v. \6: prima attestazione registrata in Manzoni).

${ }^{80}$ La loc. nasce probabilmente proprio in quegli anni: la prima accezione registrata dal GDLI (s.v. medaglione \$6) appartiene all' Appendice di B. Migliorini ('pezzo di carne tagliata in forma di disco') al Dižionario moderno di Panzini (1942).

${ }^{81}$ I traducenti medaglioni di filetto e rocchio si ritrovano già in Jàcono (1939).

82 Cfr. Piacentini (2016: 161) per la presenza del termine nel Dizionario di Panzini.
} 
A dispetto degli sforzi traduttivi, non sempre il sostituto proposto aderisce perfettamente al referente di partenza (emblematico il caso della coppia omelette-frittata, le cui differenze Bianchi non manca di rilevare, pur accettando l'uso del termine italiano) ${ }^{83}$, e ciò sarà tra i principali motivi di insuccesso delle censure linguistiche imposte dal regime. È quanto si osserva soprattutto con $i$ forestierismi per $i$ quali si propongono più corrispettivi italiani, nessuno dei quali, a volte, capace di restituire un collegamento del tutto univoco col referente originario: si pensi alla resa di un concetto astratto come comfort $(\rightarrow$ conforto, comodo, comodità, modernità), ma anche di oggetti concreti, che sono riprodotti per mezzo di iperonimi o, comunque, di traducenti che non appaiono pienamente sovrapponibili all'accezione propria del forestierismo (cotillons $\rightarrow$ doni, regali, sorprese; crêpe $\rightarrow$ focaccina, cialda, crespella; ma anche brioche $\rightarrow$ focaccetta; charlotte $\rightarrow$ crostata; potage $\rightarrow$ minestra; ecc.). Sono soprattutto alcune perifrasi descrittive, tuttavia, a perdere la relazione immediata col referente, come si vede in filettone di bue (per chateaubriand), latte sbattuto o caffe sbattuto (per frappè) e salsiccia di Francoforte (per Würstel), fino ad arrivare a perifrasi caratterizzate da uno spiccato grado di analiticità, ma di conseguenza molto deboli a fini sostitutivi ${ }^{84}$, quali zuppa di legumi minuti (per julienne), braciola di manzo in graticola (per Rostbraten) ${ }^{85}$, fetta di pan tostato (per toast). In qualche caso la perifrasi italiana è indotta da un corrispettivo sintagma o da una voce composta della lingua originaria: novo al latte (à la coque), costole di manzo (côtes de bouff), rosolio di ciliege (cherry-brandy), ecc.; solo di rado il sostituto italiano finisce per costituire una scelta più sintetica rispetto al forestierismo: en crồte $\rightarrow$ crostato, en belle vue $\rightarrow$ in addobbo, five o' clock $\rightarrow$ merenda (tra le voci del gruppo a, cfr. grill room $\rightarrow$ rosticceria e hors-d'oeuvre $\rightarrow$ antipasto).

\section{ESITI DELL'INIZIATIVA E RAPPORTI CON LE OPERE PURISTICHE ANTECEDENTI}

La percentuale delle proposte destinate ad avere successo, come si ricava da tutte le parole citate supra, è piuttosto limitata, in linea col fallimento generale che notoriamente caratterizzò tutta l'impresa di bonifica linguistica tentata dal regime. Tra le sostituzioni più incisive, alle quali i parlanti hanno riservato una futura rivincita sul forestierismo, o quantomeno un risultato di sostanziale pareggio, possiamo citare ascensore (per l'ingl. lift), dispensa (per l'ingl. office, e al cospetto dell'adattamento officio, proposto dalla Commissione), rinfresco (per il fr. buffet), pasticcini (per il fr. gateaux), cosciotto (per il fr. gigot), pompelmo (per l'ingl. grape-fruit), sala da ballo (per l'ingl. dancing, e al cospetto di sala di danze, proposto dalla Commissione ${ }^{86}$, secco (per l'ingl. dry), sformato (per il fr. flan), rosticceria (per l'ingl. grill-room), portiere (per il fr. concierge), (uovo) in camicia (per il fr. pochè), prosciutto (per l'adattamento giambone), ecc.: come è evidente, si tratta perlopiù di termini per i quali esisteva un sostituto ben aderente e diffuso già a quel tempo. Sono tuttora radicati nel lessico fondamentale, invece, forestierismi come brioche, champagne, comfort, frappé, garage, menu ecc., che nella lingua contemporanea non soffrono particolarmente la concorrenza dei possibili sostituti italiani.

In diversi casi, tuttavia, $i$ forestierismi hanno conosciuto un processo di invecchiamento più o meno vistoso, pur essendo ancora registrati nei principali dizionari

\footnotetext{
${ }^{83}$ Cfr. nota 25.

84 Cfr. Piacentini (2016: 179): «La scelta [...] di non rinunciare all'informatività (anzi se possibile di aumentarla) nella trasposizione lessicale, portò a proposte magari impeccabili da un punto di vista descrittivo, ma difficilmente vincenti in un'ottica sociolinguistica».

$85 \mathrm{Si}$ noti, peraltro, la diversa traduzione del germanismo Rostbraten rispetto all'anglicismo, pressoché corrispondente, roast-beef.

${ }^{86}$ Nel sito web del quotidiano la Repubblica (ricerca effettuata il 20.04.2021) troviamo circa 1.400 risultati di sala da ballo, ma appena 10 di sala di danza e 2 di sala di danze.
} 
dell'uso contemporanei: in Zingarelli (2022) troviamo, per es., voci come bordure, buvette, canard, consommé, cotillons, entremets, gigot e Kursaal (e, almeno nel caso di cotillons, andrà rilevato che la parziale o totale scomparsa odierna del forestierismo è stata ovviamente indotta dalla decadenza del rispettivo designatum). Tra i forestierismi considerabili come usciti senz'altro dall'uso, alla luce della loro mancata accoglienza in Zingarelli (2022) e Devoto, Oli (2022), possiamo invece ricordare anisette, beef-steak, blanc-manger, breakfast, canard, chateaubriand, cordial, côtes de bouf, en belle vue, en boîte, en croûte, five o' clock, homard, noisette ${ }^{87}$, page, peppermint, poularde, Rostbraten, timbale, zacusca, Zwieback: come si vede, si tratta per buona parte di marcati forestierismi di lusso (canard, five o' clock, homard), o di parole continuatesi in italiano grazie a una corrispettiva forma adattata; tra i forestierismi rubricati da Bianchi, ma non dalla Commissione, si riscontra la permanenza, sempre in Zingarelli (2022), delle voci aspic, bouquet, cabaret (s.v. cabarè, indicata come settentrionale nell'accezione di 'vassoio'), carrè, habitué, roast-beef, sandwich, sauté, Strudel.

Quanto ai rapporti di dipendenza con le due maggiori opere puriste degli anni '30 del Novecento, ovvero Monelli (1933) e Jàcono (1939), abbiamo infine ricavato alcune percentuali indicative di una discreta contiguità nelle scelte di sostituzione (anche se, in diversi casi, esse si mostrano piuttosto scontate: es. beef-steak $\rightarrow$ bistecca, canard $\rightarrow$ anatra, cordial $\rightarrow$ cordiale, giambone $\rightarrow$ prosciutto, lift $\rightarrow$ ascensore, restaurant $\rightarrow$ ristorante, trattoria, ecc.): delle 128 voci straniere elencate da Bianchi, infatti, 44 sono già prese in considerazione da Monelli (1933) e 108 da Jàcono (1939), ma soprattutto, dei 193 traducenti complessivamente proposti da Bianchi in luogo dei forestierismi, 52 si ritrovano già tra le pagine del Barbaro Dominio di Monelli e 97 in quelle del Diz̧onario di esotismi di Jàcono.

La presenza dei due grandi repertori puristi di fine Ottocento, Rigutini (1886) e Fanfani, Arlia (1890: $3^{\mathrm{a}}$ ediz.), al contrario, è abbastanza circoscritta, a testimonianza da un lato della prospettiva linguistica assunta da Bianchi (e, più in generale, dai lavori puristici di quegli anni: cfr. Serianni, 2011: 274), prevalentemente rivolta ai neologismi di introduzione recente, dall'altro lato a dimostrazione della natura spiccatamente settoriale di buona parte del lessico trattato, la cui sostituzione doveva risultare meno impellente in opere lessicografiche di taglio generale: se abbiamo ben visto, infatti, in Rigutini (1886) si leggono 13 dei 128 forestierismi poi chiamati in causa da Bianchi (tutti a loro volta presenti anche in Fanfani, Arlia 1890), mentre in Fanfani, Arlia (1890) il numero complessivo sale a 23.

Trascorrendo dalle parole allo stile, va almeno ricordato, in conclusione, come le note linguistiche allegate ai singoli lemmi offrano una lettura godibile e si rivelino non di rado spassose, attingendo talvolta ad aneddoti autobiografici ${ }^{88}$ e non rinunciando all'inserzione di giudizi estetici (es.: «brutto lombardismo», «brutto quanto inutile francesismo», «Bruttissima locuzione», «non è certo men bella», «parola, che suona male», ecc.) e di gusto spesso impressionistico (es.: «bettolino [...] pur avvicinandosi al corrispondente significato del vocabolo gallico, puzza di gattabuia lontano un miglio» [AINIT, 1940, 3-4: 110, s.v. buvette]; «Perché [...] si è proposti di chiamarli castagne candite? Non esiste forse l'italianissima parola marrone? Fra la castagna e il marrone vi è la stessa differenza che c'è

${ }^{87}$ In entrambi i dizionari (s.v. noisette) compare solo l'accezione di 'color nocciola'.

88 Basti l'esempio della voce mussare: «Ho conosciuto un cameriere che, avendo "travagliato" in Francia, parlava un così barbaro linguaggio da farmi ricordare una famosa descrizione di Parigi notturna: Proménasi il popolo / Francese la notte / Nel fango pietinano / Gommosi e cocotte / Guardati dai mille / Col sabre nel fodero / Sergenti di ville. Questo buffissimo garçon mi chiese una volta se dovesse far mussare lo Champagne frappato [...]» (AINIT, 7-8: 255): la descrizione di Parigi citata da Bianchi è tratta dal Giobbe. Serena concezione di Marco Balossardi, opera pseudonima di Olindo Guerrini e Corrado Ricci, che parodiava il Giobbe di Mario Rapisardi. 
(C) Italiano LinguaDue 2. 2021. E. Ventura, "Tradurre bisogna, non tradire!": le proposte di Icilio Bianchi in difesa della lingua italiana (1939-1940)

fra un pulcino e un pollastro» [AINIT, 1939, 7-8: 255, s.v. marrons glacés]). ${ }^{89}$ Gli interventi puristici di Bianchi restano, ad ogni modo, un documento degno d'interesse nell'àmbito della politica linguistica fascista, consentendo di verificare, una volta di più, come le decisioni ufficiali pubblicate di lì a poco nel Bol dalla Commissione siano debitrici, per ampie sezioni, verso lavori antecedenti a quelli dello stesso sodalizio accademico, che rappresentò soprattutto il momento culminante, finalizzato alla sintesi e alla sistemazione complessiva, di una stagione culturale destinata irrimediabilmente all'insuccesso ${ }^{90}$.

89 Per alcuni caratteri stilistici comuni alle rubriche puristiche del tempo, cfr. Quaglino (2009: 374-375) e Serianni (2011: 270-271).

90 A mo' di appendice, infine, ci limitiamo a ricordare qui in nota che, ai fascicoli 9-10: 321-323 e 11-12: 397-400 del 1940, sono destinati due ulteriori interventi linguistici, introdotti dal titolo emblematico Parliamo italiano anche negli alberghi!: a questi, che abbisognerebbero di una trattazione a parte, Bianchi affida la pubblicazione di un dizionarietto ( $\mathrm{di} 144$ forestierismi corredati, come sempre, da sue personali osservazioni: «lusingandoci così di portare un modesto contributo pratico all’italianissima iniziativa» AINIT, 1940: 321) che era stato precedentemente diffuso dalla CIT (Compagnia Italiana Turismo), e che si mostra di particolare interesse perché allarga ulteriormente i confini del lessico di viaggio, inglobando anche molte voci non trattate dal Nostro, e talvolta assenti anche negli elenchi del Bol: di nuovo, pertanto, Bianchi interviene sulle proposte di sostituzione che giungevano da altri ambienti della cultura di regime, facendo valere le proprie riflessioni personali e contestando, in più di un'occasione, le scelte suggerite da suoi contemporanei. Ci limitiamo qui a spigolare qualche voce, evidenziando anzitutto che, anche in tal caso, molte delle scelte sostitutive proposte (ancora riconducibili, per massima parte, alle riproduzioni semantiche e alle perifrasi descrittive) si ritroveranno identiche fra gli elenchi del Bol: muovendoci tra le prime lettere dell'alfabeto, osserviamo addition $\rightarrow$ conto; affiche $\rightarrow$ manifesto, avviso, affisso, cartello, cartellone (tutti e cinque i possibili sostituti approdati poi al Bol); agreement $\rightarrow$ accordo; airlined $\rightarrow$ aerodinamico; autocar $\rightarrow$ torpedone (turistico); autopullman $\rightarrow$ autosalone; booking clerk $\rightarrow$ impiegato di banco; budget $\rightarrow$ bilancio; camion $\rightarrow$ autocarro (ma anche càmion in Bol); capote $\rightarrow$ mantice, soffietto; carnet $\rightarrow$ libretto, taccuino; chauffage $\rightarrow$ riscaldamento; chauffage central $\rightarrow$ termosifone, ecc. In altri casi Bianchi propone delle soluzioni alternative a quelle della CIT: un esempio molto significativo, che sembra anche rivelare come la Commissione dovesse tenere ben presenti, tra le altre, proprio le proposte avanzate da Bianchi, è offerto dal fr. cliché, tradotto dalla CIT con matrice e incisione, ma dal Nostro con zinco, rame, legno («secondo la materia su cui è stata eseguita l'incisione, a tratto a retino»), una resa metonimica abbastanza distante dal referente; ebbene, nel Bol la Commissione indicherà come sostituti, oltre a stampo, proprio i tre traducenti promossi dal Nostro (sottintendendo stampo di...); altrove le scelte di Bianchi si riveleranno perdenti, come sarà per il fr. couchette, reso con letto («Cuccetta [...] tutt'al più può dirsi del lettuccio del marinaio») piuttosto che con cuccetta, proposto dalla CIT e poi dalla stessa Commissione. Più in generale, dagli interventi di Bianchi trapelano motivazioni che già ci è capitato di mettere in luce supra: anzitutto la necessità, quando possibile, di evitare neosemie nelle sostituzioni: così, ad es., per allottment, reso come lotto dal CIT, Bianchi osserva che il «Lotto [...] è il gioco che tutti conoscono, ed anche (ma è voce contrastata dai puristi) ciascuna delle parti in cui è diviso un tutto [...]» (AINIT, 1940, 9-10: 321), motivo per cui sarà meglio dire disponibilità o disponibili; similmente, per il fr. carte, reso dal CIT con tessera oppure carta di legittimazione (a sua volta calco del fr. carte de légitimation), proprio l'uso del determinante genererebbe un ulteriore problema di neosemia («legittimaz̧ione ha altri significati: è l'atto che attribuisce a chi è nato prima del matrimonio la qualità di figlio legittimo» AINIT, 1940,11-12: 322) e sarà perciò da preferire la loc. carta d'identità. In maniera complementare, va evitata l'adozione di traducenti riferibili a più contesti diversi, tali da indurre in confusione, come ben esemplificato dal trattamento dell'anglicismo boy, per il quale il CIT propone fattorino, ragazzo, valletto, paggio, esponendosi così alle puntualizzazioni semantiche di Bianchi («non v'ha dubbio che andiamo incontro ad un'inevitabile confusione. Valletto si adatta di più al giovane di scuderia; fattorino al ragazzo che esegue commissioni fuori dell'albergo; ragazzo o, meglio, ragazzo dell'ascensore, al lift-boy» AINIT, 1940, 11-12: 397); a sua volta, il significato dell'ingl. traveller's check andrà restituito tramite la sola loc. assegno per viaggiatori, senza ricorrere ad altri due traducenti, proposti dalla CIT ma già impiegati in differenti contesti («Sarebbe forse necessario, per evitare confusione, fermarsi alla prima definizione, l'unica esatta: assegno per viaggiatori [...]. L'assegno turistico, invece, è un assegno emesso all'estero e al cambio di favore della lira turistica [...]. L'assegno circolare, infine, è il normale assegno rilasciato dalle Banche e che non può circolare all'estero» AINIT, 1940, 11-12: 397); al contrario, poi, per l'ingl. box Bianchi rileva l'omissione di una particolare accezione, che richiederebbe l'uso dell'it. stallo, termine poi accolto anche nel Bol («la parola box è usata da noi anche per indicare lo scompartimento separato delle stalle e delle scuderie, nel qual caso va tradotta in stallo» AINIT, 1940, 9-10: 321). Diversi, anche in queste liste, sono i chiari forestierismi di lusso, la cui permanenza in italiano sembra peraltro essere stata piuttosto effimera, come nel caso del ted. Bestellung $\rightarrow$ ordinativo, 
(C) Italiano LinguaDue 2. 2021. E. Ventura, "Tradurre bisogna, non tradire!": le proposte di Icilio Bianchi in difesa della lingua italiana (1939-1940)

\section{RIFERIMENTI BIBLIOGRAFICI}

Adamo G., Della Valle V. (2008), Le parole del lessico italiano, Carocci, Roma.

Artusi P. (2010), La scienza in cucina e l'arte di mangiar bene, a cura di Capatti A., Rizzoli, Milano.

Beccaria G. L. (2012), "Varietà e unità nella lingua di Artusi”, in Frosini G., Montanari M. (a cura di), Il secolo artusiano, Atti del convegno (Firenze-Forlimpopoli, 30 Marzo-2 Aprile 2011), Accademia della Crusca, Firenze, pp. 3-5.

Capatti A. (1998), "Lingua, regioni e gastronomia dall’Unità alla seconda guerra mondiale", in Capatti A., De Bernardi A., Varni A. (a cura di), Storia d'Italia, Annali, vol. 13, L'alimentarione, Einaudi, Torino, pp. 753-801.

Capatti A., Montanari M. (2005), La cucina italiana. Storia di una cultura, Laterza, Roma-Bari. Cicioni M. (1984), 'La campagna per l'autarchia della lingua': una 'bonifica' fallita", in Parlare fascista. Lingua del fascismo, politica linguistica del fascismo, numero monografico di «Movimento operaio e socialista», VII, 1, pp. 87-95.

DELI = Cortelazzo M., Zolli P., Dirionario etimologico della lingua italiana, $2^{\mathrm{a}}$ ediz. (a cura di Cortelazzo M. e Cortelazzo M. A.), Zanichelli, Bologna, 1999.

Devoto G., Oli G. C. (2022), Nuovo Devoto-Oli. Il vocabolario dell'italiano contemporaneo 2022, a cura di Luca Serianni e Maurizio Trifone, Le Monnier, Firenze.

DI = Schweickard W., Deonomasticon Italicum. Diæ̌ionario storico dei derivati da nomi geografici e da nomi di persona, Niemeyer, Tübingen, 1997; vol. 1: Derivati da nomi geografici: A-E (1997-2002); vol. 2: Derivati da nomi geografici: F-L (2006); vol. 3: Derivati da nomi geografici: M-Q (2009); vol. 4: Derivati da nomi geografici: R-Z (2013); Supplemento bibliografico. Seconda edirione riveduta e ampliata, De Gruyter, Berlin-Boston, 2013).

Fanfani M. (2018), Un dizionario dell'era fascista, Società Editrice Fiorentina, Firenze.

Fanfani P., Arlia C. (1890), Lessico dellinfima e corrotta italianità, Paolo Carrara Editore, Milano ( $3^{\text {a }}$ ediz.).

Foresti F. (a cura di) (2003), Credere, obbedire, combattere: il regime linguistico nel Ventennio, Pendragon, Bologna.

Frosini G. (2012), "La cucina degli italiani. Tradizione e lingua dall'Italia al mondo", in Mattarucco G. (a cura di), Italiano per il mondo, Accademia della Crusca, Firenze.

GGI (1931) = Guida gastronomica d'Italia, Touring Club Italiano, Milano.

prenotazione, o degli anglicismi chef booking clerk $\rightarrow$ capobanco, deck (nave) $\rightarrow$ ponte, elevetor/lift $\rightarrow$ ascensore, overnight $\rightarrow$ pernottamento, lit-salon (voce assente nel Bol) $\rightarrow$ scompartimento con letti (di treni), ecc. Non mancano neppure, però, le parole straniere la cui traducibilità in italiano appare molto complicata, o pressoché impossibile: molto indicativo il caso di cabaret, sostituito dalla CIT con la perifrasi generica ritrovo notturno, rispetto alla quale Bianchi ammette la difficoltà di trovare un traducente migliore («per quanto la deformazione sia troppo generica, non sapremmo suggerire una miglior locuzione italiana» AINIT, 1940, 9-10: 322); un forestierismo cui viene offerta cittadinanza per mancanza di valide alternative è oblò, adattamento del fr. bublot («Per quanto trattasi di un gallicismo, è assai probabile che la parola rimarrà nell'uso. Infatti, non ci estasiamo soverchiamente al termine marinaresco occhio di bue (si dice altresì occhio di cubia) proposto dal Panzini stesso [...]. Finestrino è troppo generico. Sportello, anche sulle navi, è tutt'altra cosa» AINIT, 1940, 9-10: 397), che la CIT rendeva con le riproduzioni semantiche finestrino o sportello, e per il quale la Commissione sceglierà invece portellino. Tra i forestierismi di più largo successo vale la pena ancora menzionare, per il valore paradigmatico degli interventi puristici apportati da Bianchi, il sost. festival, per il quale la CIT propone festa e festino, mentre la Commissione sceglierà l'adattamento festivale, precedentemente già adottato da Jacono (e già per Bianchi degno di accettazione alla luce della sua provenienza dall'agg. latino festivalis): ancora una volta Bianchi manifesta l'inutilità di opporre resistenza a parole straniere difficilmente sostituibili, e lo fa senza rinunciare alle sue consuete stoccate contro certe proposte stravaganti della lessicografia precedente («in qualche caso sarebbe meglio tradurre convegno, mostra [...]. Giustamente lo Jacono propone di usare la parola Festivale [...]. Il Fanfani butta là - risum teneatis! - il vocabolo...musicone! Meglio un esotismo che una buffonata!» AINIT, 1940, 9-10: 323). 
(C) Italiano LinguaDue 2. 2021. E. Ventura, “Tradurre bisogna, non tradire!”: le proposte di Icilio Bianchi in difesa della lingua italiana (1939-1940)

GDLI = Grande Dižionario della Lingua Italiana, a cura di Battaglia S., 21 voll., UTET, Torino, 1961-2002.

Gheno V. (2020), "Di krapfen e graffe", in Italiano digitale, XIII, 2, pp. 77-80.

Jàcono A. (1939), Diæionario di esotismi, Marzocco, Firenze.

Klein G. (1986), La politica linguistica del fascismo, il Mulino, Bologna.

Latini A. (1694), Lo scalco alla moderna, overo l'arte del ben disporre i conviti..., nella nuova stampa delli socii Dom. Ant. Parrino, e Michele Luigi Mutii, Napoli.

Leso E. et al. (1976), La lingua italiana e il fascismo, Consorzio provinciale pubblica lettura, Bologna.

Mangoni L. (1974), L'interventismo della cultura: intellettuali e riviste del fascismo, Laterza, RomaBari.

Monelli P. (1933), Barbaro dominio, Hoepli, Milano.

Natali F. (1940), Come si dice in italiano? Vocabolarietto autarchico, Bergamo Fascista, Bergamo.

Nichil R. L. (2011), "«Si dispone che...». Sulla politica linguistica del fascismo, dal Foglio di disposizioni a «Lingua Nostra»", in Nesi A., Morgana S., Maraschio N. (a cura di), Storia della lingua italiana e storia dell'Italia Unita, Atti del IX Convegno ASLI (Firenze, 2-4 dicembre 2010), Franco Cesati Editore, Firenze, pp. 439-450.

Nichil R. L. (2012), "Il purismo linguistico fascista dal Foglio di disposizioni del P.N.F. al Bollettino di informazioni della Reale Accademia d'Italia", in Bianchi P. et al. (a cura di), La variazione nellitaliano e nella sua storia. Varietà e varianti linguistiche e testuali, Atti dell'XI Congresso SILFI (Napoli, 5-7 ottobre 2010), Franco Cesati Editore, Firenze, vol. 1, pp. 85-94.

Panzini A. $\left(1923^{4}\right)$, Diæionario moderno: Supplemento ai dirionari italiani, Hoepli, Milano.

Piacentini L. (2016), “«Parole nostre a casa nostra, fino all'estremo limite del possibile». Le italianizzazioni gastronomiche della Reale Accademia d'Italia (1941-1943)", in Studi di lessicografia italiana, XXXIII, pp. 151-186.

Piacentini L. (2017), "«E sì che nel mio libro deve aver spigolato a mansalva». Monelli, Jàcono e l'ipotesi di un plagio", in Studi di lessicografia italiana, XXXIV, pp. 307-323.

Pirani R., Mare M., De Antoni M. G. (2000), Dizionario Bibliografico del Giallo, Bibliografica Editrice, Pontassieve.

Quaglino M. (2009), “«E non cominceremo a trasformare il menù in saporosa godenda?». Autarchia linguistica e cucina nella stampa quotidiana e periodica (1930-1945)", in Robustelli C., Frosini G. (a cura di) (2009), Storia della lingua e storia della cucina, Atti del Convegno ASLI (Modena, 20-22 Settembre 2007), Franco Cesati Editore, Firenze, pp. 365-375.

Raffaelli A. (2008), "La deonomastica francese negli elenchi della Commissione per l'italianità della lingua (1941-1943)", in Caffarelli E., D'Achille P. (a cura di) Lessicografia e Onomastica 2, Atti del Convegno Internazionale (Roma, 14-16 febbraio 2008), Quaderni della Rivista Internazionale di Onomastica, Società Editrice Romana, Roma, pp. 337-348.

Raffaelli A. (2009), "Forestierismi e italianizzazioni di ambito gastronomico della Reale Accademia d'Italia", in Robustelli C., Frosini G. (a cura di), Storia della lingua e storia della cucina, Atti del Convegno ASLI (Modena, 20-22 Settembre 2007), Franco Cesati Editore, Firenze, pp. 349-363.

Raffaelli A. (2010a), "La lingua del fascismo", in Simone R. (a cura di), Enciclopedia dell'Italiano, Istituto dell'Enciclopedia italiana, Roma:

http://www.treccani.it/enciclopedia/lingua-del-fascismo_(Enciclopediadell'Italiano)/.

Raffaelli A. (2010b), Le parole straniere sostituite dall'Accademia d'Italia (1941-43), Aracne, Roma. 
(C) Italiano LinguaDue 2. 2021. E. Ventura, “Tradurre bisogna, non tradire!”: le proposte di Icilio Bianchi in difesa della lingua italiana (1939-1940)

Raffaelli S. (1983), Le parole proibite. Purismo di Stato e regolamentazione della pubblicità in Italia (1812-1945), il Mulino, Bologna.

Raffaelli S. (2006), "La vicenda dei neologismi a corso forzoso nell'Accademia d'Italia", in Adamo G., Della Valle V. (a cura di), Che fine fanno i neologismi? A cento anni dalla pubblicazione del Diz̧ionario moderno di Alfredo Panzini, Leo S. Olschki, Firenze, pp. 91 104.

Ricci L. (2005), La lingua dellimpero. Comunicaz̧ione, letteratura e propaganda nell'età del colonialismo italiano, Carocci, Roma.

Rigutini G. (1886), I neologismi buoni e cattivi più frequenti nell'uso odierno, Libreria Editrice Carlo Verdesi, Roma.

Scarpati C. (1979), "Le lettere di Pascoli ad Augusto Guido Bianchi", in Aevum, LIII, 3, pp. 439-497.

Serianni L. (2006), "Panzini lessicografo fra parole e cose", in Adamo G., Della Valle V. (a cura di), Che fine fanno $i$ neologismi? A cento anni dalla pubblicazione del Dizionario moderno di Alfredo Panæini, Leo S. Olschki, Firenze, pp. 55-78.

Serianni L. (2011), "Monelli, Jàcono, Silvagni: gli ultimi repertori di esotismi", in Caffarelli E., Fanfani M. (a cura di), Lo spettacolo delle parole. Studi di storia linguistica e di onomastica in ricordo di Sergio Raffaelli, Società Editrice Romana, 2011, Roma, pp. 269-282.

TLIO $=$ Tesoro della lingua Italiana delle Origini, fondato da Pietro G. Beltrami e continuato da Lino Leonardi, diretto da Paolo Squillacioti: http:/ / tlio.ovi.cnr.it/TLIO/.

Zingarelli N. (2022), Lo Zingarelli 2022. Vocabolario della lingua italiana, a cura di Cannella M., Lazzarini B., Zaninello A., Zanichelli, Bologna. 